\title{
Real-time estimation of the optically detected magnetic resonance shift in diamond quantum thermometry toward biological applications
}

\author{
Masazumi Fujiwara $\odot,{ }^{1, *}$ Alexander Dohms $\odot,{ }^{2}$ Ken Suto, ${ }^{1}$ Yushi Nishimura, ${ }^{1}$ Keisuke Oshimi,,${ }^{1}$ Yoshio Teki $\odot,{ }^{1}$ Kai Cai $\odot,{ }^{3}$ \\ Oliver Benson, ${ }^{2}$ and Yutaka Shikano ${ }^{4,5, \dagger}$ \\ ${ }^{1}$ Department of Chemistry, Osaka City University, Sumiyoshi-ku, Osaka, 558-8585, Japan \\ ${ }^{2}$ Institut für Physik und IRIS Adlershof, Humboldt Universität zu Berlin, Newtonstrasse 15, 12489 Berlin, Germany \\ ${ }^{3}$ Department of Electrical and Information Engineering, Osaka City University, Sumiyoshi-ku, Osaka, 558-8585, Japan \\ ${ }^{4}$ Quantum Computing Center, Keio University, 3-14-1 Hiyoshi, Kohoku, Yokohama, 223-8522, Japan \\ ${ }^{5}$ Institute for Quantum Studies, Chapman University, 1 University Dr., Orange, California 92866, USA
}

(Received 20 July 2020; accepted 19 November 2020; published 24 December 2020)

\begin{abstract}
Real-time estimation protocols for the frequency shift of optically detected magnetic resonance (ODMR) of nitrogen-vacancy (NV) centers in nanodiamonds (NDs) are the key to the recent demonstrations of diamond quantum thermometry inside living animals. Here we analyze the estimation process in multipoint ODMR measurement techniques (3-, 4-, and 6-point methods) and quantify the amount of measurement artifact derived from the optical power-dependent ODMR spectral shape and instrumental errors of experimental hardware. We propose a practical approach to minimize the effect of these factors, which allows for measuring accurate temperatures of single ND during dynamic thermal events. Further, we discuss integration of noise filters, data estimation protocols, and possible artifacts for further developments in real-time temperature estimation. This study provides technical details regarding quantum diamond thermometry and analyzes the factors that may affect the temperature estimation in biological applications.
\end{abstract}

DOI: 10.1103/PhysRevResearch.2.043415

\section{INTRODUCTION}

Quantum nanoscale sensing based on optically detected magnetic resonance (ODMR) of nitrogen-vacancy (NV) centers in nanodiamonds (NDs) enables highly sensitive nanoscale probing of magnetic fields, electric fields, and temperature [1-4]. Reading the frequency shift of ODMR using continuous-wave $(\mathrm{CW})$ or pulsed measurements is the fundamental step. Recent studies have focused on developing this sensing technique for practical applications [5-10] and have accordingly tried to efficiently deploy the sensors into real-time measurement systems [11-16].

For biological applications, the use for thermometry is of particular significance because temperature is a fundamental parameter of biological activity, such as circadian rhythms [17], energy metabolism [18], and developmental processes $[19,20]$. The biological application of NV thermometry was first demonstrated in cultured cells [21-24] and, recently, in in vivo model animals, such as nematode worms $[25,26]$. A technological key to the recent in vivo demonstrations is the method that can efficiently determine the temperature of

\footnotetext{
*masazumi@osaka-cu.ac.jp

†yutaka.shikano@keio.jp

Published by the American Physical Society under the terms of the Creative Commons Attribution 4.0 International license. Further distribution of this work must maintain attribution to the author(s) and the published article's title, journal citation, and DOI.
}

NDs under their dynamic motion inside biological structures; one needs to complete the temperature measurement for a few seconds because the incorporated NDs and the whole systems are moving, which significantly challenges the current quantum-sensing protocols. Among the various ODMR measurement protocols related to thermometry [21,27-34], multipoint methods have been proposed [11,21,29,32] to accelerate the ODMR measurement process; here, fluorescence intensities at three or four frequency points are acquired to estimate the ODMR shift. These methods significantly reduce the measurement time enabling more signal sampling, which improves the precision, compared with the frequency-shift determination using the whole-spectral-shape measurements. These methods, however, are inevitably susceptible to experimental errors, such as the change of ODMR spectral shape, temperature-dependent NV fluorescence intensity, and hardware instrumental errors, owing to estimate the frequency shift based on the limited available information at the selected frequency points.

Until recently, two types of multipoint ODMR methods (i.e., 3- and 4-point methods) have demonstrated their effectiveness $[21,29]$. Intuitively, the number of frequency points is likely to affect the results; the estimation based on a higher number of frequency points provides more information and presents a robust behavior under the complicated response of the ODMR spectral shape, which cannot be simplified using the simple Lorentzian function. However, increasing the number of frequency points reduces the number of measurements, which may lead to decline in precision. Thus, understanding error and noise sources associated with these multipoint 
ODMR measurements is critical to further use these techniques.

In this study, we study the multipoint ODMR measurements in the context of real-time thermometry for biological applications. We first show that there is a marginal difference in the photo-responsivity between the photon counts of the selected frequencies, which causes substantial artifacts during temperature estimations. This difference is derived from the optical power dependence of the ODMR spectral shape. In addition, hardware instrumental errors may affect the photoresponsivity depending on the selected experimental devices, which become significant in the proposed 6-point method (an extended version of the 4-point method). Second, we propose a practical method to cancel this effect and compare the performances of each method in terms of precision and associated noises. Third, we demonstrate the monitoring of the temperature dynamics of single NDs while having a dynamic variation of fluorescence intensity by the thermal drift of the setup and temperature dependence of NV fluorescence. Finally, we summarize the above-detailed investigation and analyze the possible artifacts that may be produced while using multipoint ODMR measurement techniques on biological samples. Note that this study is highly associated with our recent publication regarding real-time in vivo quantum thermometry [26], which will be referred to in several instances hereinafter.

\section{EXPERIMENTS}

\section{A. CW and multipoint ODMR measurements and external temperature control}

We used a home-built confocal microscope equipped with a microwave system for the ODMR measurements, as schematically depicted in Fig. 1 (see Appendix A for details). A CW 532-nm laser combined with an acousto-optic modulator was used for pulsing the laser illumination. The laser power was computer-controlled using a set of a half-wave plate and a polarizer. The optical excitation intensity was $\sim 2$ $\mathrm{kW} \mathrm{cm}{ }^{-2}$, unless specified. An oil-immersion objective with a numerical aperture of 1.4 was used. The NV fluorescence was filtered using a dichroic beam splitter and long-pass filters and sent to an avalanche photodiode (APD) in the confocal detection system. The output of an APD was fed to two data-acquisition (DAQ) boards equipped with four pulse counters (DAQ-1) and two counters (DAQ-2). We used NDs containing $~ 500 \mathrm{NV}$ per particle (Adámas Nanotechnologies, NDNV100nmHi10ml) that were spin coated on coverslips. The sample coverslips were set in an incubation chamber that was mounted on a piezo stage. Note that an external magnetic field was not used in this study, except for Fig. 13.

We used six individual microwave sources and an SP6T switch with a switching time of $250 \mathrm{~ns}$ for the CW and multipoint ODMR measurements (Fig. 10). The output microwave signal was amplified and fed to a microwave linear antenna (25- $\mu \mathrm{m}$-thin copper wire) placed on a coverslip that was sealed with a home-built plastic box with a hole at the center. The typical microwave excitation power was estimated to be $10-50 \mathrm{~mW}(10-17 \mathrm{dBm})$ at the linear antenna by considering the source output, amplifier gain, and the experimentally determined $S_{21}$ of the antenna system, which provides a microwave magnetic field of more than $2-5 \mathrm{G}$ in $20 \mu \mathrm{m}$ from the antenna (see Ref. [26] for more details). To acquire the CW-ODMR spectra, the APD detection was gated for the microwave irradiation ON and OFF using the SP6T switch and a bit pattern generator. Specifically, the bit-pattern generator fed TTL pulses to the SP6T switch for gating the output from MW1 (200 $\mu$ s for ON and OFF), which is followed by a $100-\mu \mathrm{s}$ TTL pulse to the acousto-optic modulator for switching off the laser, as indicated in Fig. 1(b). We prepared two counters in DAQ-1 [Sig and Ref in Fig. 1(b)] and fed the APD output to these two counters. These two counters were operated in "gated edge counting mode." This resulted in $I_{\mathrm{PL}}^{\mathrm{ON}}$ and $I_{\mathrm{PL}}^{\mathrm{OFF}}$ with a repetition rate of $2 \mathrm{kHz}$. In the multipoint-ODMR measurements, the APD detection was gated for the respective microwave frequencies ( 3 points: $\omega_{-}, \omega_{+}$, and off-resonant $\omega_{0} ; 4$ points: $\omega_{1}$ to $\omega_{4} ; 6$ points: $\omega_{1}$ to $\left.\omega_{4}, \omega_{-}, \omega_{+}\right)$. The gate width was $t_{\mathrm{M}}=100 \mu \mathrm{s}$, which was common for all three, four, or six gates, each followed by an interval of $t_{\text {int }}=5 \mu \mathrm{s}$. For the 3- or 4-point method, we used three or four counters in DAQ-1, each synchronized to the respective microwave sources [Figs. 1(c) and 1(d)]. For the 6-point method, two counters of DAQ-2 were used in addition to the four counters of DAQ-1, owing to the limited counter availability in DAQ-1 [Fig. 1(e)]. The total photon count $I_{\text {tot }}$ was obtained using the following equation:

$$
I_{\mathrm{tot}}=\frac{t_{\mathrm{M}}+t_{\mathrm{int}}}{t_{\mathrm{M}}} \times \sum_{k}^{3,4,6} I_{k} .
$$

The factor $\left(t_{\mathrm{M}}+t_{\text {int }}\right) / t_{\mathrm{M}}=1.05$ was obtained considering $t_{\mathrm{M}}=100 \mu \mathrm{s}$ and $t_{\text {int }}=5 \mu \mathrm{s}$.

During the temperature measurements, a confocal microscope system was used to track the target NDs because the external temperature change presented in Sec. IIID caused a substantial positional drift of NDs on the coverslip of more than tens of micrometers with a speed of $100 \mathrm{~nm} / \mathrm{s}$ (see Ref. [26] for the information regarding positional drift). The piezo stage was first scanned in the $x y z$ directions while measuring the ND fluorescence intensity. The obtained cross sections of the point spread function along the $x y z$ axes were fitted using Gaussian functions to determine the $x y z$ positions for repositioning. Subsequently, the stage was moved smoothly to the repositioning point in five steps of $\sim 20 \mathrm{~nm}$, every $2 \mathrm{~ms}$. The repositioning required $3.8 \mathrm{~s}$ and was performed with a tracking period of $t_{\text {track }}=4 \mathrm{~s}$, as depicted in Fig. 1(f). Data acquisition for the temperature estimation was performed for $1.0 \mathrm{~s}$ (occasionally, for $0.5 \mathrm{~s}$, depending on the application) for every $4 \mathrm{~s}$. Note that the current effective integration of positional tracking is crucial to most biological applications, as recently demonstrated in the quantum thermometry of nematode worms [26].

The sample temperature $\left(T_{\mathrm{S}}\right)$ was varied via direct heat conduction from the oil-immersion microscope objective, whose temperature $\left(T_{\mathrm{obj}}\right)$ was controlled by using a PIDfeedback controller of the foil heater that wrapped the objective. $T_{\mathrm{S}}$ was calibrated in the following manner: (1) a flat miniscule Pt100 resistance temperature probe $(5 \times 5 \times$ $0.2 \mathrm{~mm}^{3}$ ) was tightly attached to the sample coverslip by a thin layer of silicone vacuum grease between the probe and 
(a)

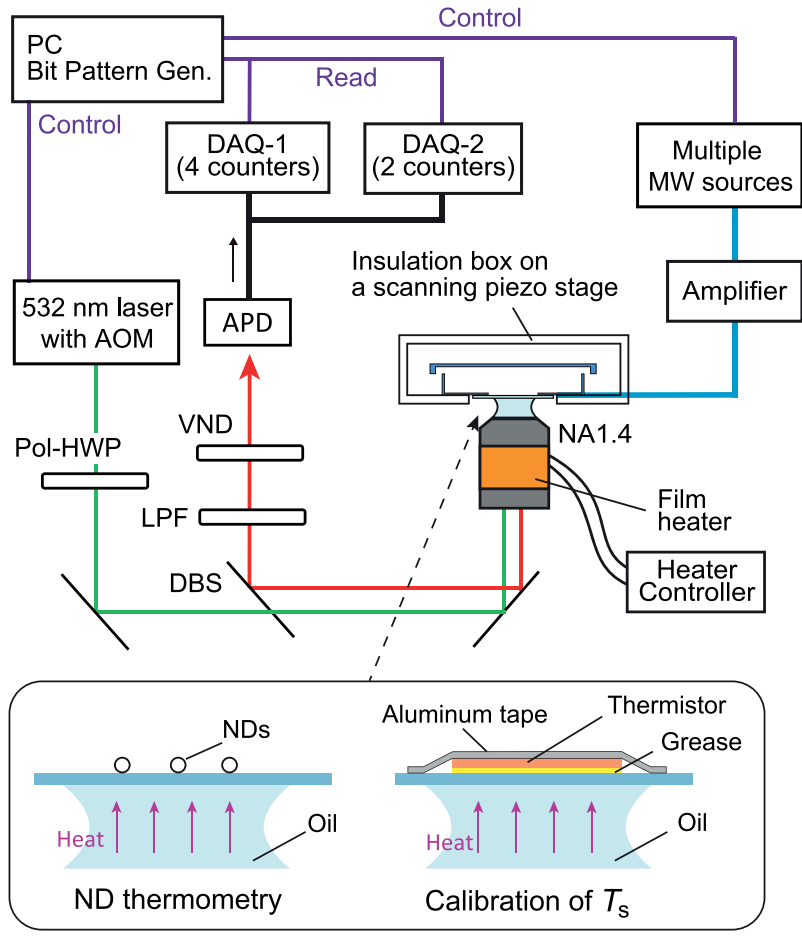

(f)

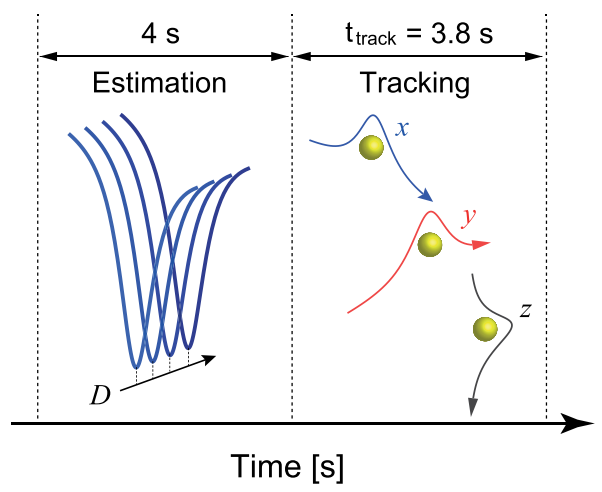

(b)

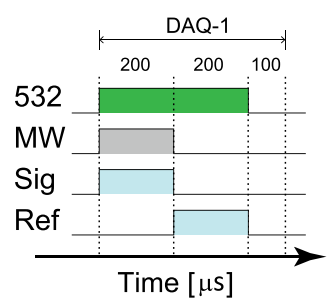

(d)

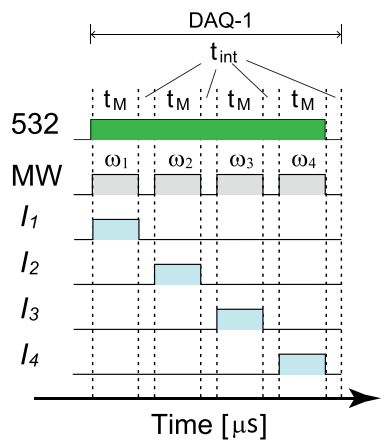

(g)

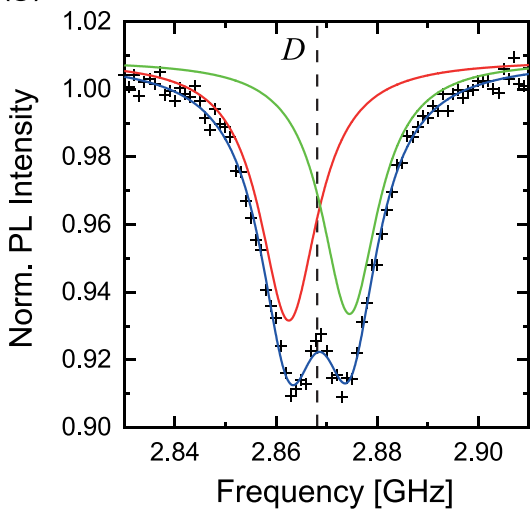

(c)

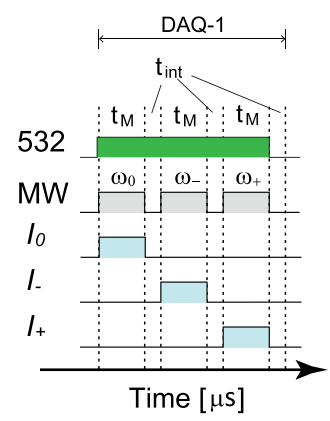

(e)

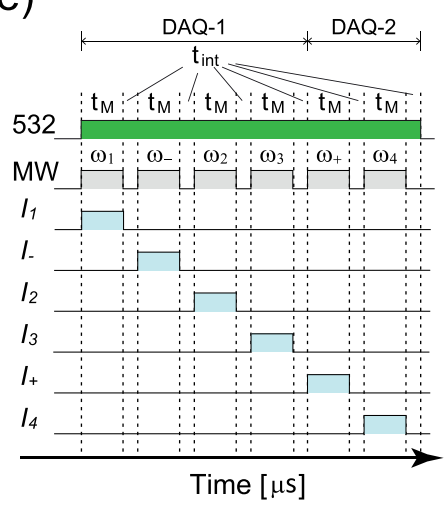

(h)

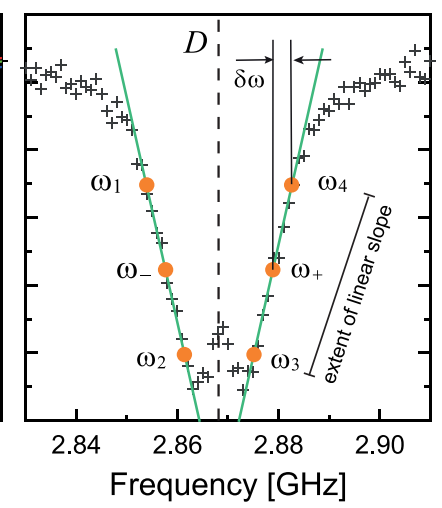

FIG. 1. (a) Schematic drawing of the experimental setup for the optical layout and microwave circuit. AOM: acousto-optic modulator. Pol-HWP: optical power adjustment unit consisting of polarizer and half-wave plate. VND: variable neutral density filter. DBS: dichroic beam splitter. LPF: long-pass filter. APD: avalanche photodiode. MW: microwave. DAQ: data-acquisition board. (Inset) Close up of NDs spin-coated on a coverslip with immersion oil and of the configuration of the thermistor attached to the coverslip for the temperature calibration of $T_{\mathrm{S}}$. (b) Pulse control sequences for CW-ODMR measurements and (c, d, e) 3-, 4-, and 6-point measurements. 532: green laser. Sig: signal for $I_{\mathrm{PL}}^{\mathrm{ON}}$. Ref: reference for $I_{\mathrm{PL}}^{\mathrm{OFF}} . \omega_{1}$ to $\omega_{4}$ are the four frequencies used for the 4-point measurements. $\omega_{-}, \omega_{+}$are the additional frequencies used for 6-point measurements. For the 3-point measurement, $\omega_{-}, \omega_{+}$and off-resonant $\omega_{0}(2.65 \mathrm{GHz})$ were used. $I_{1}$ to $I_{4}$ and $I_{-}, I_{+}$are the corresponding photon counts. $t_{\mathrm{M}}$ : measurement time. $t_{\text {int }}$ : interval time. (f) A sequence of the estimation of the zero-field splitting $(D)$ and three-dimensional particle tracking in the $x y z$ directions. $t_{\text {track }}$ : tracking period. (g) ODMR spectrum fitted using the sum of two Lorentzian functions and (h) that with two linear slopes fitted to the spectrum. The selected frequencies $\omega_{1}$ to $\omega_{4}$ and $\omega_{-}, \omega_{+}$are indicated by the orange circles from left to right and are separated on each of the slopes by $\delta \omega$, as shown in the inset. Note that $\omega_{0}=2.65 \mathrm{GHz}$ used for the 3-point method is not shown.

the coverslip [inset of Fig. 1(a)]. (2) The probe was completely covered with aluminum tape whose edges were glued to the base coverslip. (3) In this thermal configuration, $T_{\mathrm{obj}}$ was varied while monitoring $T_{\mathrm{S}}$. We obtained the following relation: $T_{\mathrm{S}}=1.847+0.923 T_{\mathrm{obj}}{ }^{\circ} \mathrm{C}$ (the calibration data can be found in Ref. [26]). The temperature probe was read using a high-precision handheld thermometer (precision: $\pm 0.02 \mathrm{~K}$ ).
During the calibration measurement, $T_{\text {air }}$ was monitored using a data logger (precision: $\pm 0.5 \mathrm{~K}$ ), and $T_{\text {air }}$ was confirmed to fluctuate within only $\pm 0.5 \mathrm{~K}$ over $12 \mathrm{~h}$. Note that $T_{\text {obj }}$ was monitored directly on top of the foil heater. The temperature stability in the incubator was $\pm 0.2^{\circ} \mathrm{C}$ over $12 \mathrm{~h}$, when measured by the above temperature probe (see Ref. [26] for the details). 


\section{B. ODMR spectral analysis to define the frequency points for the multipoint ODMR measurements}

To determine the microwave frequencies for the multipoint ODMR measurements, the CW-ODMR spectra were first recorded for the target NDs. Based on the obtained CWODMR spectra, the intensities $I_{1}$ to $I_{4}$ and $I_{-}, I_{+}$were selected in the following manner: (1) The obtained CW-ODMR spectra were fitted to the sum of two Lorentzian functions to indicate the ODMR dip and the zero-field splitting $D$, as shown in Fig. 1(g). (2) The two linear slopes of the ODMR dip were determined via linear fits and six frequency points that included three on each slope, which were uniformly distributed (i.e., equidistant with $\delta \omega$ over the extent of the slopes). In this case, $\omega_{-}$and $\omega_{+}$were centered on the zero-field splitting $(D)$ such that $I\left(\omega_{-}\right)=I\left(\omega_{+}\right)$[see Fig. 1(e) and Appendix B for the detailed procedure].

Combinations of the fluorescence intensity values at these frequency points provide the ODMR shift estimation $\left(\Delta \Omega_{\mathrm{NV}}\right)$ for the 3-, 4-, and 6-point methods. For the 3-point method, $\omega_{-}$and $\omega_{+}$were considered for the two frequencies on the Lorentzian dip, and $\omega_{0}=2.65 \mathrm{GHz}$ was set for the off-resonance frequency to obtain the baseline fluorescence intensity. The temperature estimate can then be expressed as follows [29]:

$$
\begin{aligned}
& \Delta \Omega_{3 \mathrm{pnt}}=-\Gamma \frac{1+\rho^{2}}{2 \rho} \frac{I_{+}-I_{-}}{2 I_{0}-I_{+}-I_{-}}, \\
& \Delta T_{\mathrm{NV}}^{3 \mathrm{pnt}}=\alpha^{-1} \Delta \Omega_{3 \mathrm{pnt}},
\end{aligned}
$$

where $\rho=\left|\omega_{+}-\omega_{-}\right| / \Gamma$. Here, $\Gamma$ and $\alpha=d D / d T$ are the full width at half maximum (FWHM) of a single Lorentzian and the temperature dependence of the zero-field splitting that mainly originates from thermal lattice expansion [35-38], respectively. Note that $2.65 \mathrm{GHz}$ was chosen as the off-resonant microwave frequency because it is sufficiently distant from the $2.87 \mathrm{GHz}$ resonant frequency, but it is not distant enough to change the high-frequency noise to the piezo stage or other electronic devices. For the 4-point method [21], the following is considered:

$$
\begin{aligned}
\Delta \Omega_{4 \mathrm{pnt}}^{1} & =\delta \omega \frac{\left(I_{1}+I_{2}\right)-\left(I_{3}+I_{4}\right)}{\left(I_{1}-I_{2}\right)-\left(I_{3}-I_{4}\right)}, \\
\Delta T_{\mathrm{NV}}^{4 \mathrm{pnt}} & =\alpha^{-1} \Delta \Omega_{4 \mathrm{pnt}}^{1},
\end{aligned}
$$

by assuming the same absolute value for the two linear slopes (Appendix C). For the present 6-point method, the mean of the three types of estimates of the 4-point method is obtained as follows:

$$
\begin{aligned}
& \Delta \Omega_{4 \mathrm{pnt}}^{1}=\delta \omega \frac{\left(I_{1}+I_{2}\right)-\left(I_{3}+I_{4}\right)}{\left(I_{1}-I_{2}\right)-\left(I_{3}-I_{4}\right)}, \\
& \Delta \Omega_{4 \mathrm{pnt}}^{2}=\frac{\delta \omega}{2} \frac{\left(I_{1}+I_{-}\right)-\left(I_{+}+I_{4}\right)}{\left(I_{1}-I_{-}\right)-\left(I_{+}-I_{4}\right)}, \\
& \Delta \Omega_{4 \mathrm{pnt}}^{3}=\frac{\delta \omega}{2} \frac{\left(I_{-}+I_{2}\right)-\left(I_{3}+I_{+}\right)}{\left(I_{-}-I_{2}\right)-\left(I_{3}-I_{+}\right)} .
\end{aligned}
$$

Then, their mean is determined as follows:

$$
\begin{aligned}
& \Delta \Omega_{6 \mathrm{pnt}}=\frac{\Delta \Omega_{4 \mathrm{pnt}}^{1}+\Delta \Omega_{4 \mathrm{pnt}}^{2}+\Delta \Omega_{4 \mathrm{pnt}}^{3}}{3}, \\
& \Delta T_{\mathrm{NV}}^{6 \mathrm{pnt}}=\alpha^{-1} \Delta \Omega_{6 \mathrm{pnt}} .
\end{aligned}
$$

Note that a comparison of the precision of these multipoint ODMR methods needs experimental validation, which is theoretically complex. Single sequences of the 3-, 4-, and 6-point measurements use $t_{3 \text { pnt }}=315 \mu \mathrm{s}, t_{4 \text { pnt }}=420 \mu \mathrm{s}$ and $t_{6 \mathrm{pnt}}=630 \mu \mathrm{s}$, respectively. Assuming the photon count rate is $R$, the photon shot noise of a single measurement of the $i$-point method $(\sigma)$ can be expressed as $\sqrt{R \times t_{i \mathrm{pnt}}}$. The measurement noise with a 1 -s sampling time is determined by the number of measurements per second $\left(t_{i \text { pnt }}^{-1}\right)$, which provides the measurement noise

$$
\sigma_{i \mathrm{pnt}}=\sqrt{R} \times t_{i \mathrm{pnt}} .
$$

Therefore, the 3-point method can provide the smallest precision in a simplistic picture because it can integrate as many measurements (estimation) as possible for a certain time. However, there are other factors affecting the precision of the thermometry, such as the spectral shape of the ODMR. The assumption of a single Lorentzian shape is not straightforward because an ODMR dip exhibits splitting due to the crystal strain and the mixed contribution from the interference between the ${ }^{3} \mathrm{~A}$ spin states and inhomogeneous decoherence sources $[22,39,40]$. This spectral shape dependency will be analyzed in Sec. III A in detail. The 4- and 6-point methods do not explicitly use such an assumption of the spectral shape; however, the selection of frequency points affects the measurement. For example, frequency points on one side $\left(\omega_{1}\right.$ and $\omega_{2}$ or $\omega_{3}$ and $\left.\omega_{4}\right)$ should be apart from each other to obtain smaller noise $\left(\sim \sigma_{4 \text { pnt }} / \delta \omega\right)$. However, they need to be sufficiently far from the curved region of the ODMR spectrum, where the linear fit is no longer valid.

\section{Experimental determination of the precision and accuracy}

The precision $\left(\sigma_{\exp }\right)$ of the thermometry was experimentally determined by using the standard errors of 20 sampling points of $\Delta T_{\mathrm{NV}}$ that were recorded over $38 \mathrm{~s}$, including tracking time. The sensitivity $\left(\eta_{T}\right)$ could be calculated as $\eta_{T}=\sigma_{\exp } \times \sqrt{2 \delta t_{\text {intgr }}}$ because this duration comprises a 19.4$\mathrm{s}$ integration time $\left(\delta t_{\text {intgr }}\right)$ and a 18.6 -s repositioning time. The accuracy was determined by adding an offset $\left(T_{0}\right)$ to $\Delta T_{\mathrm{NV}}$ to match $T_{\mathrm{S}}$ and obtaining the root mean square (RMS) of $T_{\mathrm{S}}-\left(T_{0}+\Delta T_{\mathrm{NV}}\right)$. The upper bound of the RMS in the steady state was considered to be the accuracy. Note that the fluctuation of the environmental temperature $T_{\text {air }}$ (namely, air-conditioned laboratory room temperature) caused a deviation of $T_{0}+\Delta T_{\mathrm{NV}}$ from $T_{\mathrm{S}}$, which overestimated the accuracy value.

\section{RESULTS AND DISCUSSIONS}

\section{A. Optical power dependency of ODMR spectra and the resultant systematic errors in the quantum thermometry}

In the multipoint ODMR methods, the counter values on each side of the CW-ODMR spectrum should present the same 
(a)

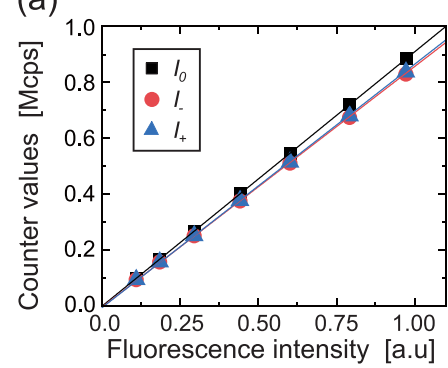

(b)

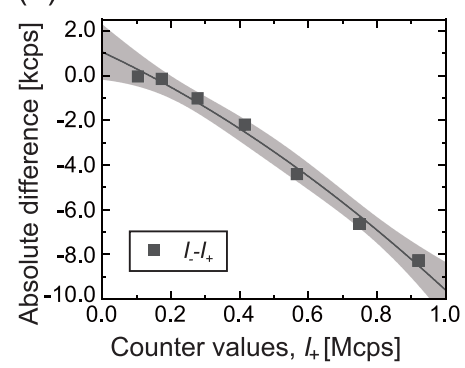

(c)

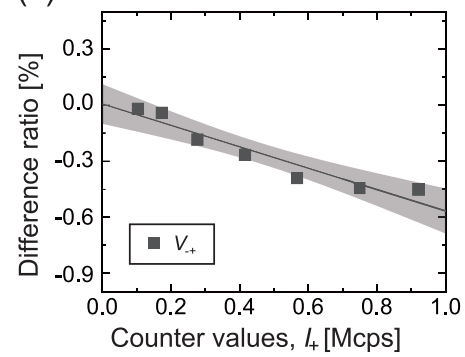

(d)

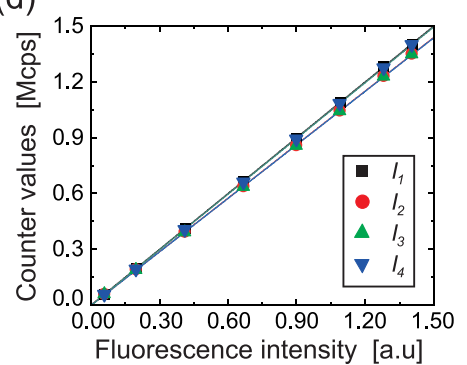

(e)

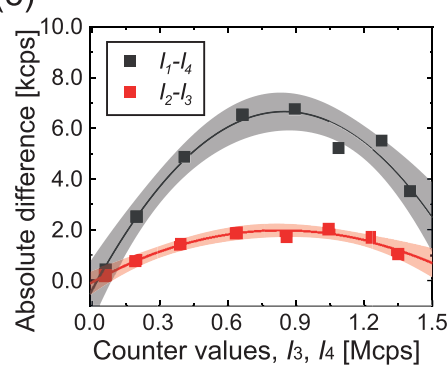

(f)

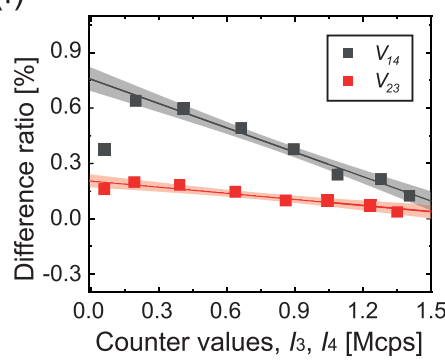

(g)

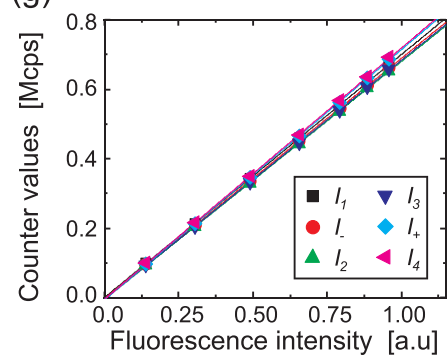

(h)

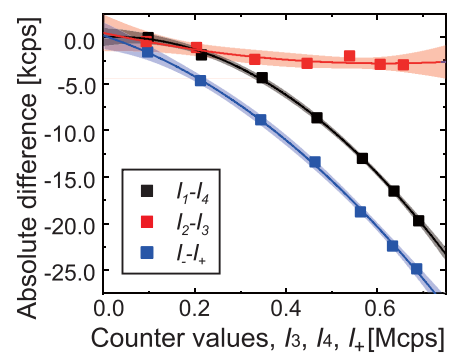

(i)

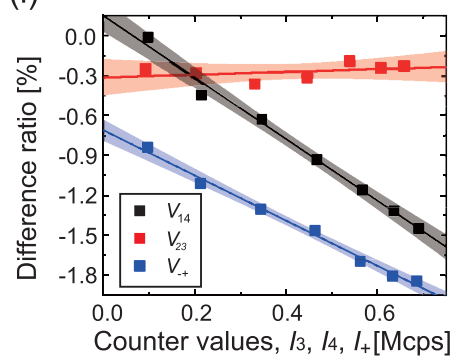

FIG. 2. Variation of the photo-responsivity of the counters. (a, d, g) Photon counts for each counter as a function of ND fluorescence with their linear fits for the 3-, 4-, and 6-point measurements, respectively. (b, e, h) Difference in the counter values between the sets of two counters, namely, $I_{-}-I_{+}$as a function of $I_{-}$for the 3-point, $I_{1}-I_{4}, I_{2}-I_{3}$ as functions of $I_{3}$ and $I_{4}$ for the 4-point and $I_{1}-I_{4}, I_{2}-I_{3}, I_{-}-I_{+}$ as functions of $I_{3}, I_{4}$, and $I_{-}$for the 6-point measurements, respectively. The solid lines and shaded area indicate second-order polynomial fits to the data and their $95 \%$ confidence intervals, respectively. (c, f, and i) The corresponding difference ratio of $V_{i j}$. The solid lines and shaded area indicate linear fits to the data and their $95 \%$ confidence intervals, respectively.

dependency on the fluorescence counts in principle; however, it was found that they presented a slightly different dependency. Figure 2(a) presents the dependence of the counter values as a function of the fluorescence intensity for the 3-point measurement, where the fluorescence intensity was varied by controlling the laser excitation power [specifically using Pol-HWP in Fig. 1(a)]. First, the counter value of $I_{0}$ is always larger than those of $I_{-}$and $I_{+}$because they exhibit the difference of the ODMR contrast. Second, $I_{-}$and $I_{+}$ increase with slightly different slopes on the order of $10^{-3}$. Figures 2(b) and 2(c), respectively, present the difference of these two counter values $\left(I_{-}-I_{+}\right)$and their ratio to the absolute counts that are defined as follows:

$$
V_{i j}=\frac{I_{i}-I_{j}}{I_{i}+I_{j}}
$$

where $i$ and $j$ denote the counter identification numbers. The absolute differences can be fitted with the second-order polynomials, and $V_{i j}$ exhibits a linear variation. The observed small variations of $V_{i j}$ of approximately $0.5 \%$ is significant in the temperature estimation process, which corresponds to a temperature variation of a few Kelvin.
The monotonic decrease of $V_{i j}$ and its resultant absolute differences of the photo-responsivity can also be observed for the 4-point measurement [Figs. 2(d)-2(f)]. For the 6-point measurement, the variations of $V_{i j}$ increase up to $\sim 2.0 \%$, as shown in Figs. 2(g)-2(i). In particular, $V_{14}$ and $V_{-+}$gave more variations (and similar to each other) than $V_{23}$ that still keeps the same level with that of the 4-point measurement. As indicated in Sec. III B, the 6-point measurement data include prominent instrumental factors, such as different clock speeds of DAQ boards. These results indicate that there should be an optical-power-dependent variation of the ODMR spectra on the order of $0.5 \%$, and additional instrumental artifacts may exist depending on the methods and apparatus used.

To better understand this photo-responsivity, the optical power dependence of the ODMR spectra was measured. Because the expected spectral variation was less than $0.5 \%$, a number of data sets were accumulated to obtain the sufficient signal-to-noise ratio $(\mathrm{SNR}=50)$. Figure 3(a) shows a set of ODMR spectra at different optical excitation intensities ranging from 0.6 to $6.3 \mathrm{~kW} \mathrm{~cm}^{-2}$. As the intensity increases, the ODMR contrast increases from $4 \%$ to $6 \%$ until $3.5 \mathrm{~kW} \mathrm{~cm}^{-2}$ and then gradually starts to decrease to $5.5 \%$. To quantify the 
(a)

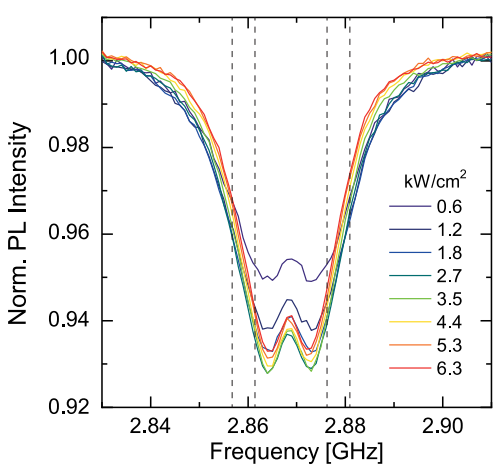

(d)

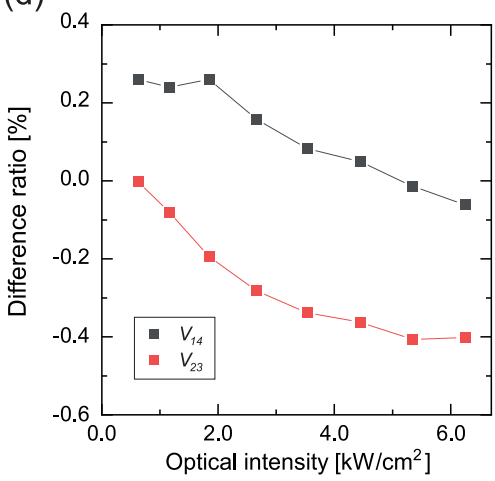

(b)

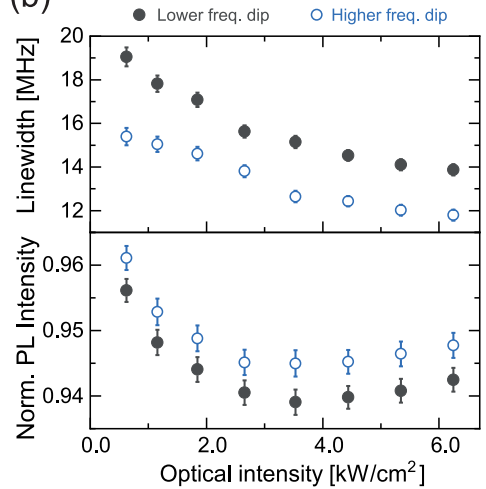

(e)

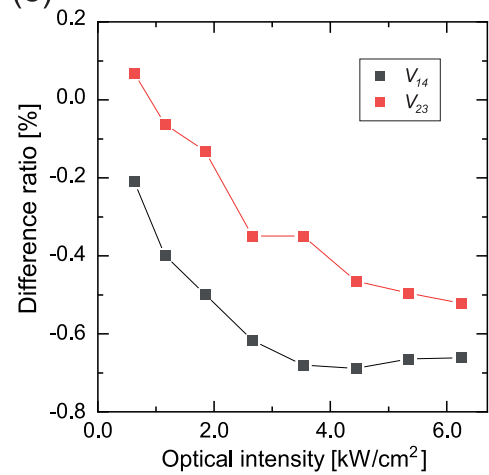

(c)

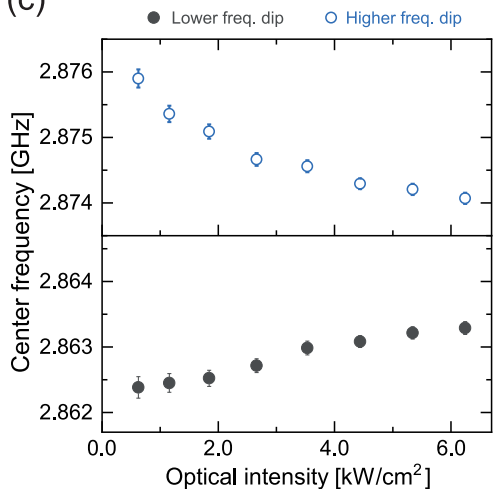

(f)

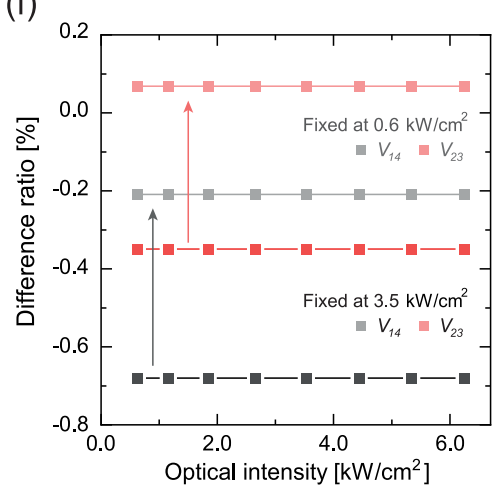

FIG. 3. Optical-power dependence of the ODMR spectra and the associated variations of $V_{14}$ and $V_{23}$ in the 4-point methods. (a) ODMR spectra for different optical excitation intensities. The optical-power dependencies of linewidth (b, top), dip area (b, bottom), and center frequencies (c) of the curve-fitted Lorentzian functions for the two splitting dips. (d) Profiles of $V_{14}$ and $V_{23}$ experimentally measured in the 4-point method for the same ND and (e) corresponding profiles of $V_{14}$ and $V_{23}$ calculated from the ODMR spectra. (f) Computed profiles of $V_{14}$ and $V_{23}$ by assuming that the Lorentzian parameters (linewidth, area, center frequency) are constant at 0.6 or $3.5 \mathrm{~kW} \mathrm{~cm} \mathrm{cor}^{-2}$ for optical power variation. The solid lines connecting the plots are a visual guide.

spectral variation, the two-dip Lorentzian fit was applied to the respective ODMR spectrum to extract the characteristic parameters, including the linewidth and contrast. Figures 3(b) and 3(c) provide the optical dependence of the linewidth, contrast, and center frequencies of the two dips. Both dips monotonically decrease the linewidth as the intensity increases, while the contrast presents a minima at $3.5 \mathrm{~kW} \mathrm{~cm}{ }^{-2}$. The center frequency exhibits a striking contrast; the lower frequency dip shifts upward in frequency by $+0.8 \mathrm{MHz}$, and the higher frequency shifts downward by $-2 \mathrm{MHz}$.

This variation causes the photo-responsivity of $V_{i j}$ in the multipoint measurements. Figures 3(d) and 3(e) present $V_{i j}$ experimentally measured in the 4-point method and computed from the ODMR spectra, respectively. Note that $V_{i j}$ decreases by $0.5 \%$ in both of figures. The $V_{i j}$ variation was confirmed to vanish when the fitting parameters of the two Lorentzian functions are fixed; in other words, no spectral variation occurs [Fig. 3(f)]. These results indicate that the photo-responsivity variation of $V_{i j}$ originate from the optical power dependence of the ODMR spectra. Note that there is an offset in $V_{i j}$, particularly in $V_{14}$, which is shifted to lower than $V_{23}$ in Fig. 3,(e) whereas it is higher in Fig. 3(e). The slight difference in the microwave power for each frequency in the multipoint methods is one of the reasons for this offset. In particular, the transmission loss for MW1 is slightly larger than MW2-MW6 owing to the difference of the cable length in our setup, which may cause the positive offset in $V_{14}$. In addition, the data presented in Figs. 3(d) and 3(e) were acquired in substantially different time scales by a factor of $300(2 \mathrm{~min}$ and $570 \mathrm{~min}$ ), making it difficult to quantitatively compare the results. More specifically, the microwave frequency was swept 20 times with a 100 -ms photon detection for single frequency points to record the single ODMR spectrum, which required $\sim 4$ min. This ODMR measurement was performed by varying the optical power (32 $\mathrm{min})$; the optical power dependence measurement was repeated 17 times in this particular experiment, for a total of $9.5 \mathrm{~h}$, including the time for manual operation. The long integration time inevitably incorporates low-frequency noise, which may affect the spectral shape.

While the exact mechanism of this optical power dependency is currently unclear, it may be due to a combined effect of the optical power dependence of ODMR in the low photon regime and the spin mixing among the electronic and nuclear spin states. The optical power dependence of the ODMR spectra was analyzed for single NV centers in Ref. [41], and the approximated model equations successfully express the power dependence of single NV centers in the optical excitation intensity range of $s>0.05$, where $s$ is the ratio of the excitation intensity to the saturation intensity of the NV centers. In this study, measurements were performed at $s<0.06$, as the saturation intensity was previously determined as $\sim 100 \mathrm{~kW} \mathrm{~cm}^{-2}$ using single NV centers [42]; in this low-photon regime, 
(a)

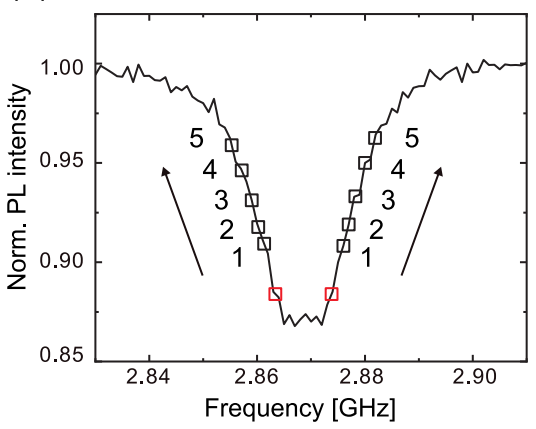

(d)

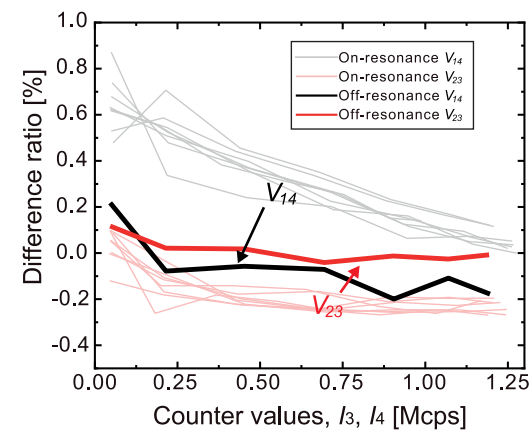

(b)

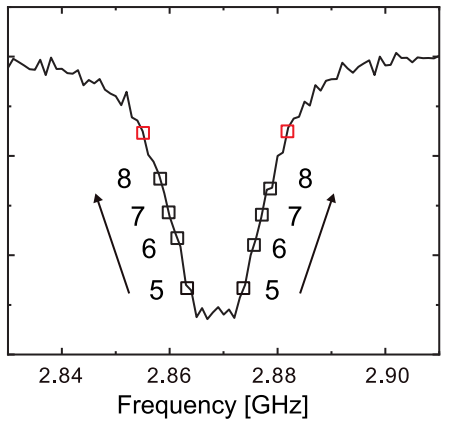

(e)

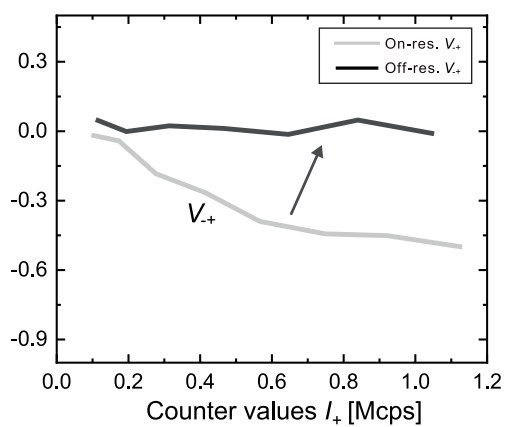

(c)

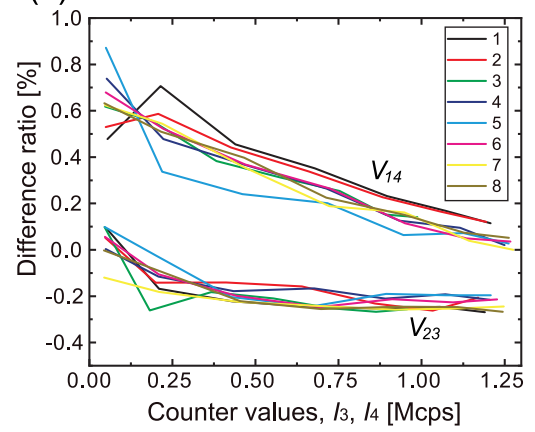

(f)

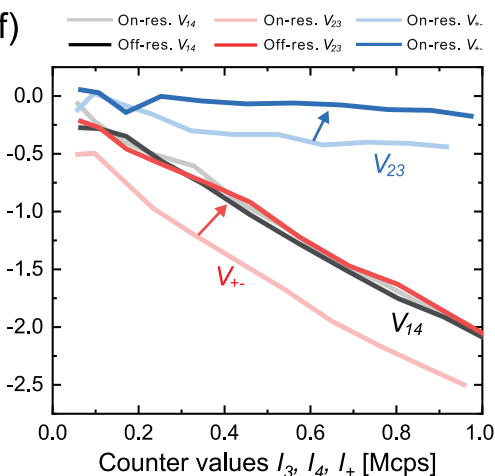

FIG. 4. Effect of the measurement frequencies to the variation of the photo-responsivity. (a) CW-ODMR spectrum with selected frequencies for the 4-point case; the two bottom frequencies (red) were fixed, and the two upper frequencies (black) were varied to be distant from the bottom two in order of $\mathbf{1} \rightarrow \mathbf{5}$. (b) CW-ODMR spectrum with four selected frequencies; the two top frequencies were fixed (red), and the two lower frequencies (black) were varied to approach the top two in order of $\mathbf{5} \rightarrow \mathbf{8}$. (c) The difference ratio of $V_{14}$ and $V_{23}$ for the eight patterns of the 4-point measurements. (d) Comparison of the on-resonant eight patterns of $V_{14}, V_{23}$, and their off-resonance profiles exciting around 2.65 GHz for the 4-point measurement. (e) Comparison of the on-resonant $V_{-+}$and its off-resonance for the 3-point measurement. (f) Comparison of the on-resonant $V_{14}, V_{23}$, and $V_{-+}$and their off-resonance for the 6-point measurement.

their nonapproximated equations can still provide a reasonable dependency of the linewidth and contrast. The linewidth monotonically decreases, and the contrast has a minima at approximately $3.5 \mathrm{~kW}$ (Fig. 12 in Appendix D). This behavior is the result of the interplay between the intrinsic decoherence of the NV spin systems $\left(\Gamma_{1} \sim 1 \mathrm{kHz}, \Gamma_{2}^{*} \sim 0.1 \mathrm{MHz}\right)$ and the comparably small optical decoherence $\left(\Gamma_{c} \sim 3 \mathrm{MHz}\right)$. Furthermore, the strain-induced transverse magnetic field causes spin mixing among the electronic and nuclear spin states. The present optical dependency is strongly diminished under the presence of an external magnetic field of $32 \mathrm{G}$ (Fig. 13 in Appendix D), indicating that this energy splitting is sufficient to eliminate the effect of physical interactions (probably hyperfine interactions) causing the frequency shift. For example, Clevenson et al. reported that the spin mixing among the electronic and nuclear spin states caused the NV hyperfine level anticrossing under weak transverse magnetic fields of $\sim 3 \mathrm{G}$ [43]. The NDs used in this study usually exhibited a splitting of $\sim 10 \mathrm{MHz}$, corresponding to the transverse magnetic field of $3.6 \mathrm{G}$; the NV hyperfine level anticrossing may contribute. In addition, in a study regarding level anticrossing in higher magnetic fields, the linewidth and contrast of ODMR were substantially affected by the level anticrossing [44]. Further studies regarding the aforementioned are necessary to clarify the origin of this low-photon specific optical power dependency of the ODMR spectra.

\section{B. Quantification of the instrument-derived photo-responsivity differences}

While the observed photo-responsivity differences are mainly caused by the optical power dependence of the NV spin systems, the contribution from the instrumental systematic errors need to be quantified because the result of the 6-point method showed a different $V_{i j}$ optical dependence from that of the 3- and 4-point methods. In particular, we analyzed the effects of (1) microwave irradiation, (2) accuracy of DAQ boards, and (3) pulse sequences. We first determine the impact of selecting the microwave frequency on the $V_{i j}$ variation. Figures 4(a) and 4(b) present eight patterns of choosing frequencies in the 4-point method. In Fig. 4(a), the upper two frequency points were shifted up, away from the bottom two fixed points, thus increasing the difference of their ODMR depth in the order of $\mathbf{1} \rightarrow \mathbf{5}$. Conversely, in Fig. 4(b), the bottom two frequency points were shifted up toward the top two fixed points $(\mathbf{5} \rightarrow \mathbf{8})$. Considering these eight patterns of frequency selections in the 4-point method, the behavior of the $V_{i j}$ optical power dependence was analyzed. Figure 4(c) shows $V_{14}$ and $V_{23}$ for the eight respective patterns. The $V_{i j}$ curves are not affected by the frequency selection within the error of $V_{i j}$. In contrast, Fig. 4(d) presents the comparison of $V_{i j}$ between these eight patterns and the off-resonant pattern in which the 4-point measurement was performed by simply shifting the pattern 3 by $200 \mathrm{MHz}$ to the lower frequency (namely, 
centering at $2.65 \mathrm{GHz}$ ). The $V_{i j}$ values decrease within the range of $\pm 0.1 \%$. As shown in Fig. 4(e), this on/off-resonance behavior of the 4-point measurement is also observed in the 3-point measurement. In the 6-point measurement, shown in Fig. 4(f), $V_{23}$ exhibits the same on/off-resonance behavior as $V_{23}$ of the 4-point measurement, whereas the difference between $V_{14}$ and $V_{-+}$disappears and is exactly matching each other. This fact indicates that the use of different DAQ boards created the same dependency for $V_{14}$ and $V_{-+}$. Note that the nonlinearity in the photo-responsivity of APDs does not cause additional variation of $V_{i j} . V_{i j}$ was measured in the 4-point method for the ON and OFF positions of the microwave to determine the effect of the APD nonlinearity, as shown in Fig. 14 (Appendix E). The variations in $V_{i j}$ when the microwave is OFF are similar to the off-resonant microwave excitation presented in Fig. 4(d), indicating the APD nonlinearity is negligible.

Second, we check whether the accuracy of the counters in the DAQ boards affects the photo-responsivity differences by testing the behavior of the counter values for the constant TTL pulse trains generated by a frequency generator (see Fig. 10; frequency accuracy, $\pm 5 \mathrm{ppm}$ ). The output from the frequency generator was connected to the two DAQ-boards instead of the APD, as shown in Fig. 10 (Appendix A). Figure 5 shows the pulse-responsivity of the counter values, their differences, and $V_{i j}$ for the 4-point and 6-point measurements. Note that the photo-responsivity of the 4-point and 3-point measurements are essentially the same, and only the 4-point and 6-point measurements are compared. As expected, all the counter values show the same linear increases [Figs. 5(a) and 5(d)], in contrast to the real photon counting of ODMR, as in Figs. 2(a) and $2(\mathrm{~g})$. The slope of the linear increase is 1.005 for all the counters, which is slightly different from the ideal value of 1. This difference is, however, not critical in the multipoint ODMR measurements because the uniform effect of all counters is finally canceled, as shown in Eqs. (3)-(5). Instead, the difference between the counter values in Figs. 5(b) and 5(e) is one or two orders of magnitude smaller than that of the real photon counting shown in Figs. 2(b) and 2(e) (0-300 ppm of $V_{i j}$ compared to $0-1.8 \%$ of the photon counting). Furthermore, $V_{i j}$ is flat over the entire range with an offset and the distribution of $<300 \mathrm{ppm}$ and $\pm 50 \mathrm{ppm}$, respectively. Further, it does not indicate a significant dependence on the counter values, which can be explained by the timing accuracy of the $50 \mathrm{ppm}$ DAQ boards based on the manufacturer's specification sheet. Note that the impedance mismatch between the APD output $(50 \Omega)$ and the DAQ inputs $(1 \mathrm{k} \Omega)$ is not likely to affect the $V_{i j}$ variation. An impedance mismatch causes reflections in the coaxial electrical line and may affect the detected pulse numbers. However, the pulse counter experiments using the frequency generator (Fig. 5) also have an output impedance of $50 \Omega$, which is the same as that of APD. If the impedance mismatch causes the observed photo-responsivity difference, it should have been observed in Fig. 5.

Note that there may be other combinations of experimental devices for using the multipoint methods, which may affect the interpretation of the artifact owing to the experimental hardware. For example, an arbitrary wave-form generator could be used to make multipoint sequences, instead of using both microwave switches and several microwave frequency (a)

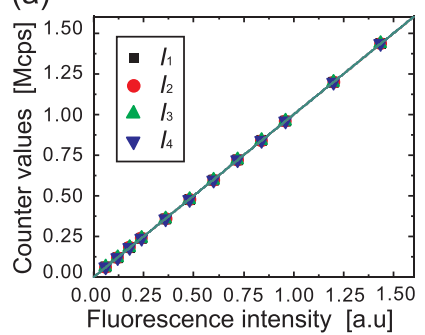

(d)

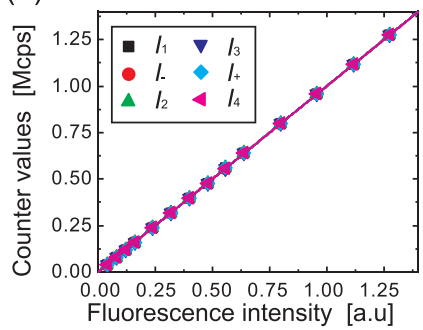

(b)

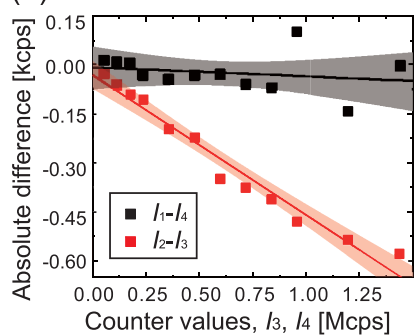

(e)

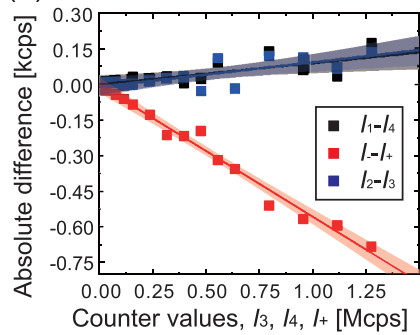

(c)

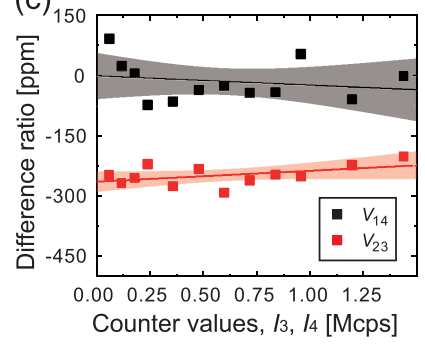

(f)

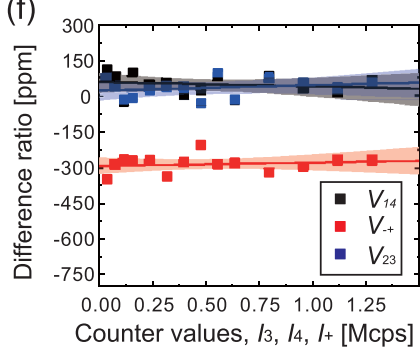

FIG. 5. Variation of counter responsivity to the constant TTL pulses from the function generator for 4- and 6-point measurements. (a,d) Total pulse counts of the four counters as a function of the input pulse counts for the 4- and 6-point measurements. (b, e) Difference between the counter values for the 4- and 6-point measurements. The solid lines and shaded area indicate second-order polynomial fits to the data and their $95 \%$ confidence intervals, respectively. (c, f) Difference in ratios for the 4- and 6-point measurements. The solid lines and shaded area indicate linear fits to the data and their $95 \%$ confidence intervals, respectively.

sources. Devising the gate-feeding to the DAQ board may reduce the number of counters used in the DAQ boards by sharing the same counters for the multiple measurement windows. Field-programmable gate array may provide more options for the implementation of multipoint ODMR. Implementation this could eliminate some of the hardware-derived measurement artifacts. In the same manner, the gated counting with a time window of $100 \mu \mathrm{s}$ used in this study may not be necessary depending on the purpose of measurement, such as when NDs do not present a noticeable positional drift. However, in this study, the present measurement system and parameters were selected because (1) they could provide rapid microwave switching that can eliminate the fast noises and (2) two DAQ boards were available at hand.

Third, the pulse sequences for the laser and microwave were further tested to determine if it caused this photoresponsivity difference by measuring its dependence on the duration of the photon-counting $\left(t_{M}\right)$ and interval times $\left(t_{\text {int }}\right)$. Figure 6(a) presents the dependence of the difference ratio acquired using the 4-point method on the measurement time, 

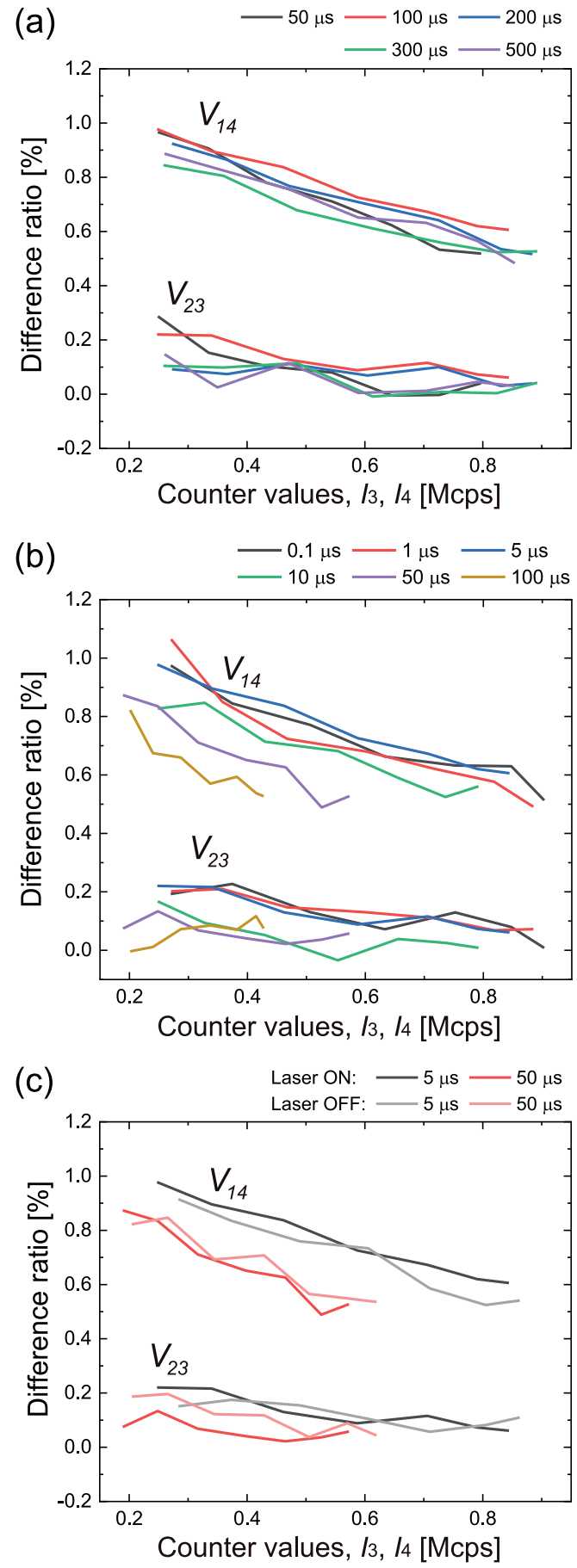

FIG. 6. Dependence of difference ratio, $V_{14}, V_{23}$, on the photoncounting measurement time, $t_{M}$ (a) and interval time, $t_{\text {int }}$ (b). (c) Difference ratio with $t_{\text {int }}=5$ and $50 \mu \mathrm{s}$ when the laser is ON or OFF during the time interval.

$t_{M}$. The dependence of $V_{14}$ and $V_{23}$ does not change significantly when the measurement time is increased from 50 to $500 \mu$ s. Similarly, Fig. 6(b) presents the dependence on the time interval $\left(t_{\text {int }}\right)$ when $t_{\text {int }}$ is increased from 0.1 to $100 \mu \mathrm{s}$. As the interval time increases, $V_{14}$ is shifted to the left (particularly 50 and $100 \mu \mathrm{s}$ ) owing to the reduction of the detected photon counts per second. However, the overall change of the difference ratio between the minimum and maximum counter values does not change because the optical power is retained at the same level. Furthermore, the laser irradiation during the interval time does not affect the photo-responsivity difference, as shown in Fig. 6(c). Note that the photo-responsivity difference is also observed in the permuted 4-point method that has been recently reported [25].

Summarizing the characterizations of the experimental hardware, the random nature of the photon-counting events only increases the measurement accuracy of the DAQ boards, and it does not cause a photo-responsivity difference of $V_{i j}$. Although using different DAQ counting boards causes a photo-responsivity difference by some unknown mechanism, it can be identified by checking the photo-responsivity difference between the on/off-resonance behaviors. A comparison of $V_{i j}$ between the off-resonant and on-resonant microwave excitation [Figs. 4(d) and 4(e)] indicates that the instrumentderived $V_{i j}$ variation is smaller than $10 \%$. The remaining $90 \%$ of $V_{i j}$ variation is, thus, contributed from the optical power dependence of the ODMR spectra.

\section{Practical approach for canceling instrumental errors}

These effects can be eliminated by subtracting preknown systematic errors from the original counter values. An errorcorrection filter is necessary particularly for the measurements operated in the low-photon regime ( $\left.I_{\text {tot }}<1 \mathrm{Mcps}\right)$, because it can create significant artifacts in the frequency-shift estimate (i.e., approximately $300 \mathrm{kHz}$ corresponding to several Kelvin). While conducting the experiments, the counter responsivity is measured each time before performing the multipoint ODMR measurements. After acquiring the counter responsivity data as presented in Fig. 2, the data were fitted with second-order polynomials, and the original counter values can be corrected as follows:

$$
\begin{aligned}
& I_{3}^{\mathrm{C}}=I_{3}^{\mathrm{NC}}+\left[a_{0}+a_{1} I_{3}^{\mathrm{NC}}+a_{2}\left(I_{3}^{\mathrm{NC}}\right)^{2}\right], \\
& I_{4}^{\mathrm{C}}=I_{4}^{\mathrm{NC}}+\left[b_{0}+b_{1} I_{4}^{\mathrm{NC}}+b_{2}\left(I_{4}^{\mathrm{NC}}\right)^{2}\right], \\
& I_{+}^{\mathrm{C}}=I_{+}^{\mathrm{NC}}+\left[c_{0}+c_{1} I_{+}^{\mathrm{NC}}+c_{2}\left(I_{+}^{\mathrm{NC}}\right)^{2}\right],
\end{aligned}
$$

where $I_{i}^{\mathrm{C}}, I_{i}^{\mathrm{NC}}, a_{k}, b_{k}$, and $c_{k}$ denote the error-corrected photon counts, original photon counts (no error correction), and coefficients of the second-order polynomials for $I_{3}, I_{4}, I_{+}$, respectively. Table I summarizes these fitting parameters used in Fig. 2. In this manner, the systematic errors of the measurement systems included in the original counter values can be eliminated. Note that this error correction does not explicitly depend on $I_{1}, I_{2}$, or $I_{-}$; the data points located on the left side of the ODMR spectrum are used concomitantly for the calculation in Eqs. (3)-(5). This independence of the other side of the ODMR spectrum is important for isolating the error correction from the temperature-derived ODMR shift.

Figure 7(a) shows the time profiles of $I_{\text {tot }}$ and $\Delta \Omega_{3 \text { pnt }}$, with and without the error correction for the 3-point measurement when the laser intensity was intentionally varied. The error correction suppresses the signal drift. While the temperature estimate shows an offset drift when the laser intensity is changed without the error correction, it no longer shows the drift when the error is corrected. It also works sufficiently when the fluorescence intensity is varied for a constant laser 
TABLE I. Fitting parameters of the second-order polynomials.

\begin{tabular}{lccr}
\hline \hline Parameters & 3-point & 4-point & 6-point \\
\hline$a_{0}+\delta a_{0}$ & - & $(-3.78 \pm 4.83) \times 10^{2}$ & $(0.27 \pm 3.57) \times 10^{2}$ \\
$a_{1}+\delta a_{1}$ & - & $(1.66 \pm 0.16) \times 10^{-2}$ & $(-2.23 \pm 2.09) \times 10^{-3}$ \\
$a_{2}+\delta a_{2}$ & - & $(-9.81 \pm 1.09) \times 10^{-9}$ & $(-4.45 \pm 0.26) \times 10^{-8}$ \\
$b_{0}+\delta b_{0}$ & - & $(-0.74 \pm 1.74) \times 10^{2}$ & $(1.80 \pm 5.19) \times 10^{2}$ \\
$b_{1}+\delta b_{1}$ & - & $(0.49 \pm 0.06) \times 10^{-2}$ & $(-1.58 \pm 0.31) \times 10^{-2}$ \\
$b_{2}+\delta b_{2}$ & - & $(-2.92 \pm 0.42) \times 10^{-9}$ & $(-3.08 \pm 0.38) \times 10^{-8}$ \\
$c_{0}+\delta c_{0}$ & $(1.05 \pm 0.45) \times 10^{3}$ & - & $(4.77 \pm 7.10) \times 10^{2}$ \\
$c_{1}+\delta c_{1}$ & $(-7.19 \pm 2.18) \times 10^{-3}$ & - & $(-1.07 \pm 0.44) \times 10^{-2}$ \\
$c_{2}+\delta c_{2}$ & $(-3.47 \pm 2.11) \times 10^{-9}$ & - & $(8.69 \pm 5.64) \times 10^{-9}$ \\
\hline \hline
\end{tabular}

intensity, as shown in Fig. 8(b); more specifically, a variable ND filter [VND in Fig. 1(a)] was varied to directly control the fluorescence intensity detected by the APD. Note that the error correction does not work sufficiently in the low- photon regime $I_{\text {tot }}<0.5 \mathrm{Mcps}$, owing to the inaccuracy of the fitting parameters. Therefore, it is necessary to always perform measurements at $I_{\text {tot }}>0.5 \mathrm{Mcps}$ to ensure that drift is negligible. (a)

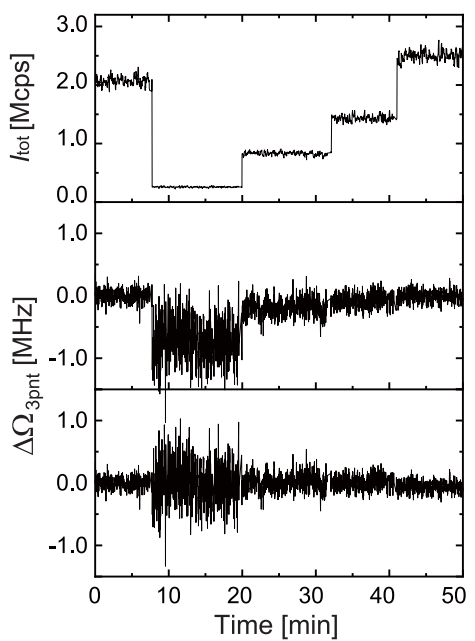

(b)

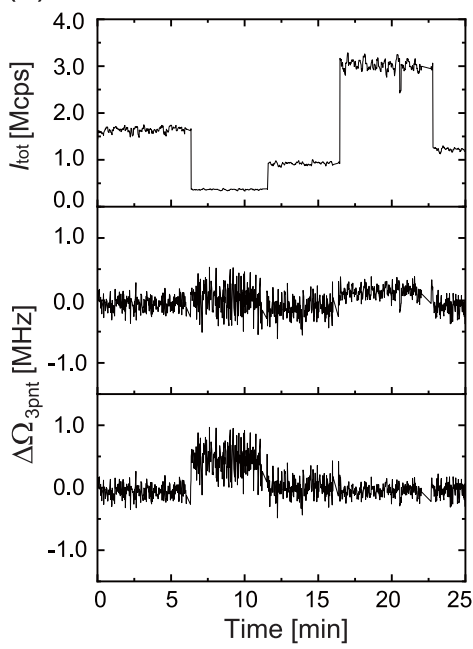

(c)

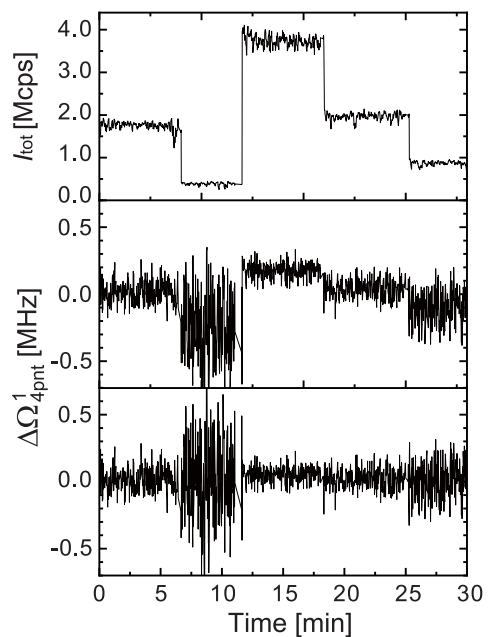

(d)

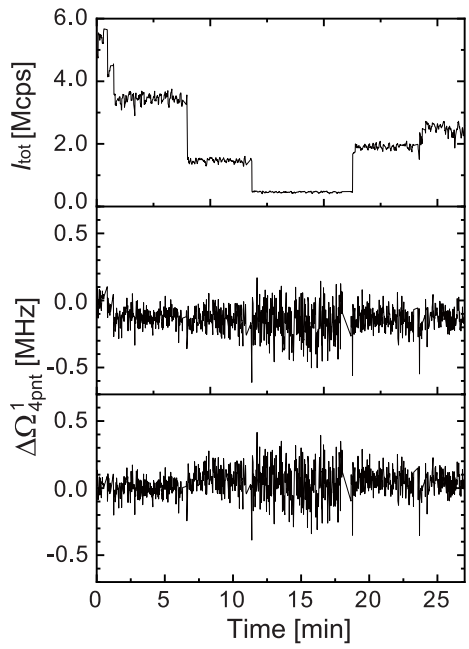

(e)

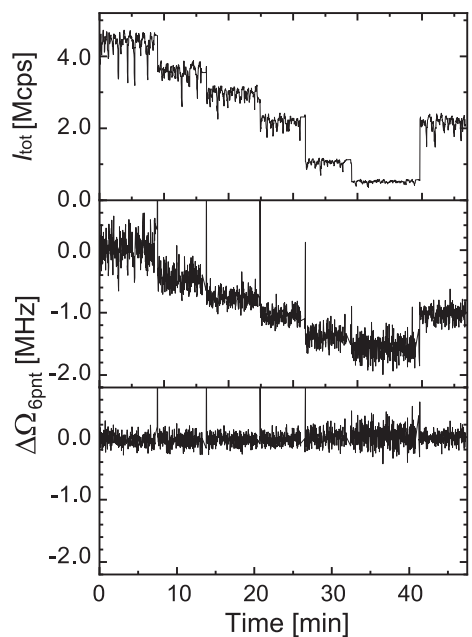

(f)

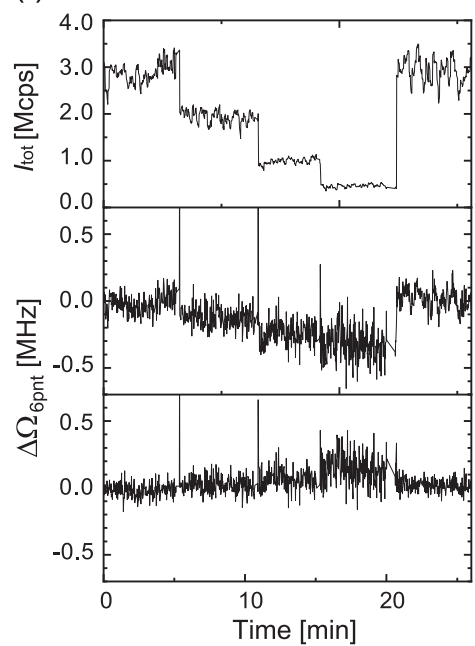

FIG. 7. Time profiles of $I_{\text {tot }}$ (top), $\Delta \Omega_{3 \text { pnt }}$ without (middle) and with the error correction (bottom) in the 3-point measurement for the cases of the laser intensity variation (a) and the PL intensity variation, respectively (b). Time profiles of $I_{\text {tot }}$ (top), $\Delta \Omega_{4 \text { pnt }}^{1}$ without (middle) and with the error correction (bottom) in the 4-point measurement for the two cases of the laser intensity variation (c) and the PL intensity variation (d). Time profiles of $I_{\text {tot }}$ (top), $\Delta \Omega_{6 \text { pnt }}$ without (middle) and with the error correction (bottom) in the 6-point measurement for the cases of the laser intensity variation (e) and the PL intensity variation (f). 
(a)

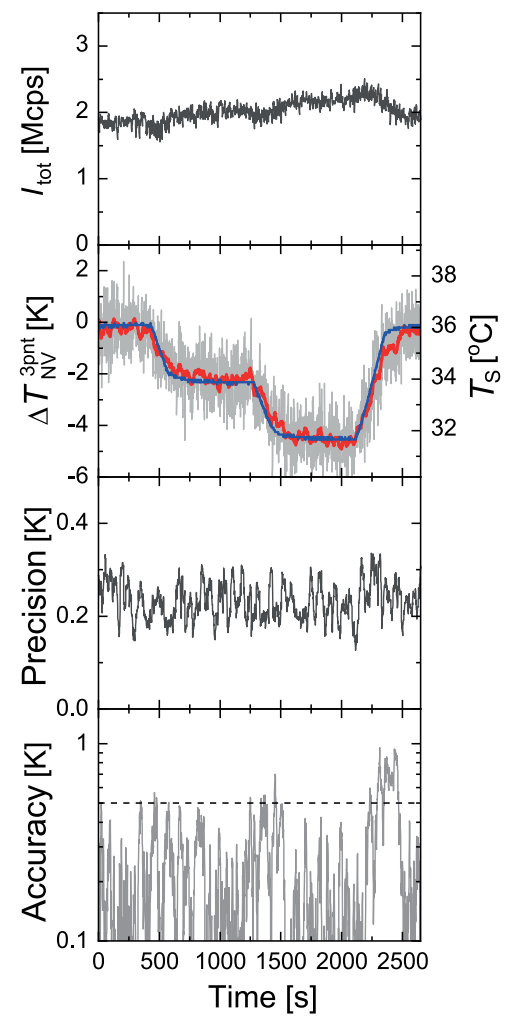

(b)

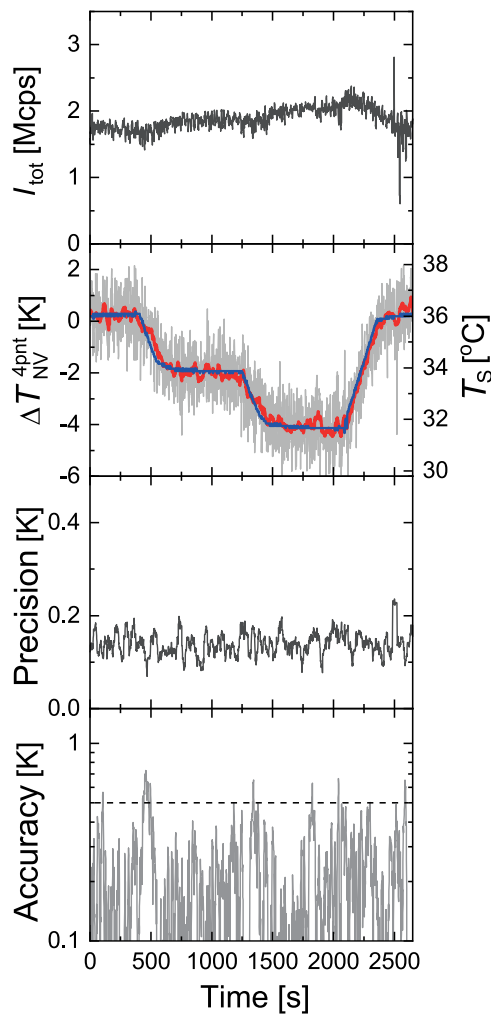

(c)

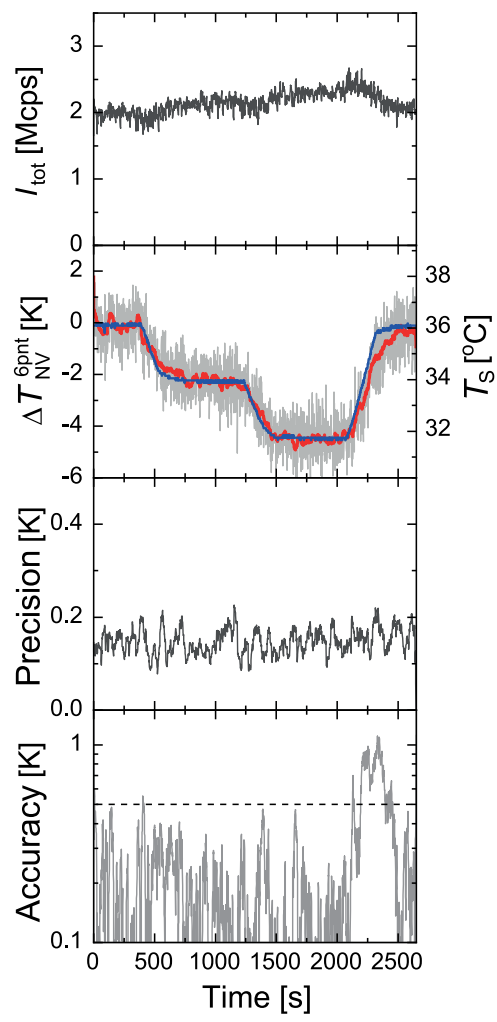

FIG. 8. Time profiles of $I_{\mathrm{tot}}$ (top), $\Delta T_{\mathrm{NV}}$ (second top), precision (second bottom), and accuracy (bottom) for the 3-point (a), 4-point (b), and 6-point measurements (c), measured on the same ND sequentially. In the second top panel, gray indicates the 1-s sampling data, red indicates the 20-point moving average, and blue indicates the sample temperature $T_{\mathrm{S}}$.

Figures 7(c)-7(f) show the corresponding data for the 4and 6-point measurements when either the laser intensity or fluorescence intensity was changed. The error correction remains effective for the 4- and 6-point measurements. For all cases, the noise is generally reduced owing to the error correction in addition to the drift cancellation. Note that the fitting errors are propagated to the absolute accuracy of the temperature measurement [Eqs. (F2)-(F3) are the error-propagation equation]. Figure 15 in Appendix F presents the dependence of the propagated errors on the fluorescence intensity for the three types of multipoint ODMR methods presented in this study. For the 3- and 4-point methods, the errors of the absolute measurement accuracy range from $0-3 \mathrm{~K}$ for most of the photon count range and can be increased up to $6 \mathrm{~K}$ when the photon counts are decreased. For the 6-point method, the accuracy is increased to 6-12 K owing to the large instrumental errors of the DAQ devices. Note that this inaccuracy indicates that there is a constant uncertain offset in the measured $\Delta T_{\mathrm{NV}}$ because the error-correction parameters are unchanged during the temperature measurement.

\section{Measuring stepwise temperature change using the multipoint methods}

Using these error corrections, the temperature change of NDs can be measured while the photon counts are dynamically changed, similar to the heating/cooling of the oil-immersion objective, which has been technically challenging because the change of the objective temperature substantially shifts the focal position, which causes a photon count noise.

Figures $8(a)-8(\mathrm{c})$ present the time profiles of the temperature estimates for the three types of the multipoint ODMR measurements by working on the same single ND with the constant optical and microwave power in the 3-, 4-, and 6-point methods, respectively. Each measurement requires approximately $40 \mathrm{~min}$ and was performed sequentially, thereby considering $\sim 2 \mathrm{~h}$ throughout these three measurements. As the temperature of the sample $\left(T_{\mathrm{S}}\right)$ is decreased stepwise by $\sim 2 \mathrm{~K}, I_{\text {tot }}$ increases (top panel). As shown in the second top panels, all the multipoint ODMR methods successfully follow the stepwise temperature change. The scaling factor between $T_{\mathrm{S}}$ and $\Delta T_{\mathrm{NV}}^{i \text { pnt }}$ varies among the three methods, providing different temperature dependencies of zero-field splitting $\alpha\left(-95.0,-54.1,-67.5 \mathrm{kHz} \mathrm{K}^{-1}\right.$ for the 3-, 4-, and 6-point measurements, respectively), while working on the same single ND. The difference between the 4- and 6point measurements originates from the fact that the $\Delta \Omega_{\mathrm{NV}}^{6 \mathrm{pnt}-3}$ (probing the deepest region of the dip) deviates from the other measurements of $\Delta \Omega_{\mathrm{NV}}^{6 \mathrm{pnt}-1}$ and $\Delta \Omega_{\mathrm{NV}}^{6 \mathrm{pnt}-2}$ (Appendix $\mathrm{G}$ and Fig. 16). This deviation indicates that the near-dip region shifted more than the base region above the FWHM, revealing a slight distortion of the spectral shape under the temperature change. The relatively large $\alpha$ of the 3 -point method is related to the assumption of the single Lorentzian shape for the ODMR spectrum. In most cases, the ODMR spectrum cannot be simplified as a single Lorentzian, and there must 
be a deviation from the Lorentzian-based estimation in the real measurements. As indicated in Fig. 17 (Appendix H), Eq. (2) holds only when the ODMR spectrum matches the single Lorentzian. Otherwise, it provides a deviation of the estimation from the real temperature change by a factor of up to 2 , depending on the real profile. We note that, in the experiment conducted in this study, the observed values of $\alpha$ (particularly of 4- and 6-point methods) are generally smaller than what was previously reported, $-74 \mathrm{kHz} \mathrm{K}^{-1}$ [35-38], which may be owing to the heating method (i.e., the NDs are heated from the bottom and cooled by the surrounding air). There is also a material inhomogeneity of $\alpha$ for both, bulk diamonds and NDs [26,35,45]. However, the present comparison between the multipoint ODMR methods is not affected by the difference of the absolute values.

The precision and accuracy of the NV thermometry during the measurement are also presented in the two bottom panels in Fig. 8. The 3-point method provides a precision of $0.23 \mathrm{~K}$, while the 4- and 6-point methods provide a slightly better precision of 0.14 and $0.15 \mathrm{~K}$, respectively. The sensitivity is then calculated as $1.4,0.9$, and $0.9 \mathrm{~K} / \sqrt{\mathrm{Hz}}$ for the $3-$, 4-, and 6-point methods, respectively, for this particular ND. The accuracy is not affected by the measurement methods and is $<0.5 \mathrm{~K}$, which is common to all the methods. Note that the accuracy presented in this study is overestimated because $\Delta T_{\mathrm{NV}}$ in the stable states is significantly influenced by environmental temperature fluctuation $\left(T_{\text {air }}\right) ; T_{\mathrm{S}}$ exhibited this effect by a $0.2 \mathrm{~K}$ fluctuation [26]. The potential accuracy of the NV thermometry should be better than the present value.

The presently used moving average of $\Delta T_{\mathrm{NV}}^{4 \text { pnt }}$ over 20 data points can track the dynamic change of the temperature. The selection of the averaging range requires careful consideration of the sensor noise (as indicated in the following section of the Allan variance analysis) and time resolution. While the 20-point moving average is optimal for the present data, other filtering techniques could be used for applications to achieve temporal resolution and temperature precision simultaneously, such as the Kalman filter (Fig. 20 in Appendix I), because these filtering techniques can work more efficiently in situations involving transient dynamics with noisy measurements [46].

Note that the temperature dependence of $I_{\text {tot }}$ is approximately 10 times larger than the estimation based on the temperature dependence of the NV fluorescence quantum efficiency. In Ref. [47], a 10\%-20\% fluorescence change was reported as the temperature increased from 27 to $77^{\circ} \mathrm{C}$, whereas a $20 \%$ fluorescence change was observed for 32 to $36{ }^{\circ} \mathrm{C}$. To determine the reason for this discrepancy, we characterized the temperature variation of the refractive index of the immersion oil as shown in Fig. 21 in Appendix J. The results indicate the refractive index of the Olympus immersion oil varies from 1.517 to 1.515 for the temperature change from 25 to $30^{\circ} \mathrm{C}$. To estimate the change of $I_{\text {tot }}$ for this temperature change, we consider that the change of the pupil size at the back focal plane of the objective is converted to the photon count change per unit area at the detector. With this simple consideration, the change of $\delta I_{\text {tot }}=\left[n\left(T_{2}\right) / n\left(T_{1}\right)\right]^{2} \sim 0.3 \%$ is calculated for the change from $T_{1}=25$ to $T_{2}=30^{\circ} \mathrm{C}$, which still remains to be substantially smaller than the actual observation. We also characterized the point spread functions
(PSFs) in the present setup at different temperatures and found that the PSF was elongated along the optical axis as the temperature increased (see Fig. 22 in Appendix J), which may explain the temperature dependence of $I_{\text {tot }}$. However, we were not able to determine if this PSF change caused the temperature dependence because of the substantial noise included in the visualized PSFs and uncertainty of the exact size of spatial filtering at the face of optical fiber working as pinhole. More detailed analysis will be necessary to explain the discrepancy between the present temperature dependence of $I_{\text {tot }}$ and the previous reports.

\section{E. Noise analysis of the ND quantum thermometry}

The noise profiles of the thermometry are analyzed next by recording long-term temperature time profiles in the respective multipoint ODMR methods. Figures 9(a) and 9(b) show the time profiles of $\Delta T_{\mathrm{NV}}^{3 \mathrm{pnt}}$ over $4 \mathrm{~h}$ and its Allan variance for the 3-point measurement, respectively. This measurement was performed at a constant temperature of $T_{\mathrm{obj}}=37^{\circ} \mathrm{C}$ $\left(T_{S}=36^{\circ} \mathrm{C}\right)$. The Allan variance profile shows four regions; it first shows a linear decrease with the slope of -0.5 until $40 \mathrm{~s}$, which indicates white noise (Region 1). Between 40 and $200 \mathrm{~s}$, the slope of the linear decrease turns by a relatively smaller value of -0.35 (Region 2, Fig. 23, Appendix K). Subsequently, the profile lands on a plateau lasting from 200 to $800 \mathrm{~s}$ (Region 3), followed by a linear increase beyond $800 \mathrm{~s}$ (Region 4). Regions 3 and 4 are mostly related to the temperature instability of the environment. Region 3 exhibits the temperature instability of the incubation chamber for the time scale of minutes. Region 4 corresponds to the air-conditioning temperature fluctuation with a period of $\sim 20 \mathrm{~min}$. Region 4 occasionally includes the instability of the NV spin properties; for example, Fig. 9(a) shows a bumpy profile while there were no noticeable changes in room temperature. These long-term fluctuations are occasionally observed in the experiments of the ND quantum thermometry, which may be related to the NV stability under the optical excitation. Currently, the noise source of Region 2 has not been clarified; however, it may be related to the mechanical instability of the confocal microscope. Therefore, the time window of the moving average filter is optimal at approximately $40 \mathrm{~s}$, corresponding to 20 adjacent points.

Figures 9(c)-9(f) show the corresponding long-term profiles of $\Delta T_{\mathrm{NV}}$ and their Allan variance profiles for the 3and 6-point measurements. Both present the common four regions in the Allan variance profiles, while there are slight differences in the prominence of Regions 2 and 4. Region 4 in the 6-point measurement further reduces in this figure; however, it increases once again beyond $1500 \mathrm{~s}$, as shown in Fig. 24(c) (Appendix K). Note that the first plateau of the noise profile until $\sim 4 \mathrm{~s}$ is due to the uneven time spacing of the data in the Allan variance calculation or the interpolation effect, as indicated in Appendix K. Furthermore, note that the tracking time can be varied as long as the stable fluorescence can be obtained; in this experiment, $t_{\text {track }}$ can be extended up to $16 \mathrm{~s}$, as shown in Fig. 25 in Appendix K.

\section{F. Artifact analysis for the temperature estimation}

Thus far, we have analyzed the systematic errors of the present thermometry system when estimating the temperature 
(a)

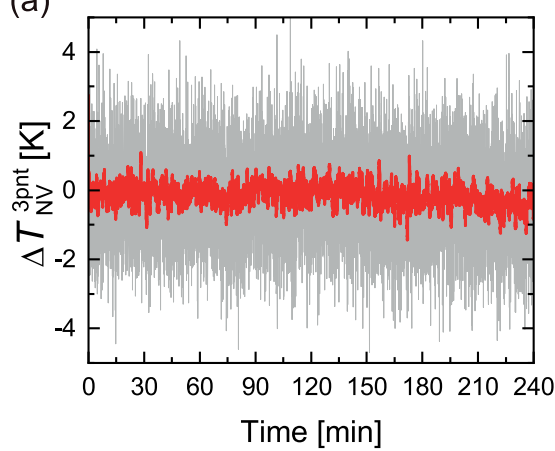

(b)

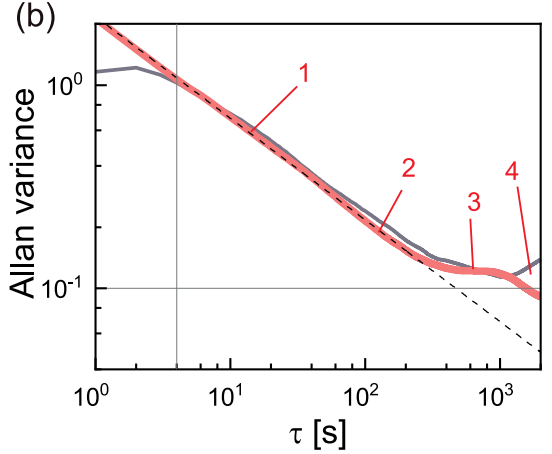

(c)

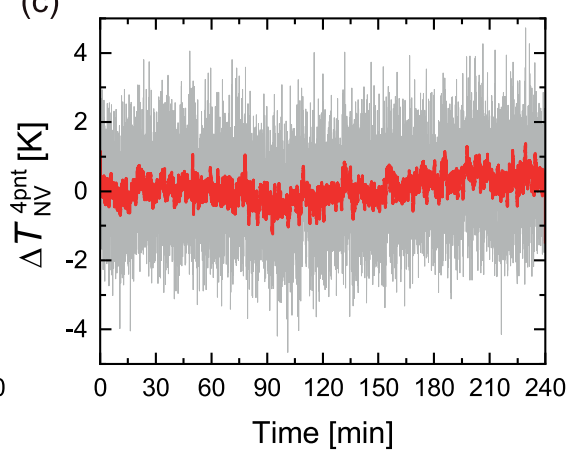

(d)

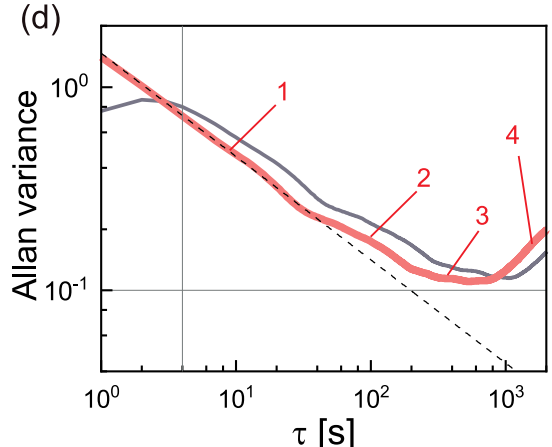

(e)

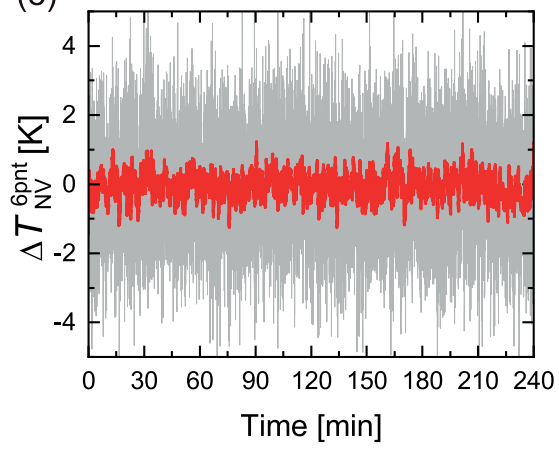

(f)

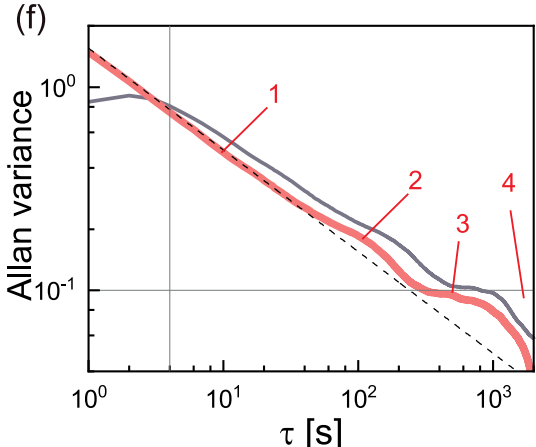

FIG. 9. Time profiles of $\Delta T_{\mathrm{NV}}$ for the 3-point (a), 4-point (c), and 6-point measurements (e). Corresponding Allan variance curves for the 3-point (b), 4-point (d), and 6-point measurements (f). The thick red line indicates the Allan variance assuming that the temperature is measured every $1 \mathrm{~s}$, regardless of the tracking time. The gray line indicates the Allan variance in the real-time scale, including the tracking time (the profile is obtained by interpolating the original data). The vertical straight line at $\tau=4 \mathrm{~s}$ indicates the tracking period. The black dotted lines indicate the linear line with the slope of -0.5 .

based on the limited available information of the ODMR spectrum. This study highlights the importance of analyzing all the possible factors that may affect the frequency shift estimation. Signals from the NV quantum thermometers may be more complicated in biochemical environments including nanofiber structures [48], lipids [49,50], cells [21,22,51], and living animals [25,26,52-54], which inevitably increases the possibility of sensory artifacts of NV centers. Table II summarizes the factors that may cause a frequency shift, linewidth change, and fluorescence intensity, which ultimately can appear as artifacts in the multipoint ODMR measurements.

The most direct factors are the magnetic field, stress, temperature, and electric field, which have been the major sensing targets of the diamond NV sensors [2]. The magnetic field

TABLE II. Real and artifact factors that can cause ODMR shift of NV centers.

\begin{tabular}{|c|c|c|c|c|c|}
\hline Source & $\begin{array}{l}\text { Physical mechanism } \\
\text { (Artifact source) }\end{array}$ & $\begin{array}{l}\text { ODMR } \\
\text { shift }\end{array}$ & Linewidth & $\begin{array}{l}\text { Photon } \\
\text { count }\end{array}$ & Ref. \\
\hline Magnetic-field (diagonal) & Zeeman & Yes & No & No & [1] \\
\hline Magnetic-field (transverse) & Spin mixing & Yes & Yes & Yes & {$[43,44]$} \\
\hline Stress & Jahn-Teller & Yes & No & No & [56] \\
\hline Temperature & Lattice-expansion (stress) & Yes & No & No & {$[21,35]$} \\
\hline Electric field & Stark & Yes & No & No & [57] \\
\hline $\begin{array}{l}\mathrm{pH} \text {, ions, } \\
\text { water adsorption }\end{array}$ & $\begin{array}{l}\text { Charge state } \\
\text { fluctuation }\end{array}$ & $\begin{array}{l}\text { Maybe } \\
\text { No }\end{array}$ & $\begin{array}{l}\text { Maybe } \\
\text { No }\end{array}$ & Yes & {$[24,64-66]$} \\
\hline $\begin{array}{l}\text { Biomolecule } \\
\text { adsorption }\end{array}$ & $\begin{array}{l}\text { Various } \\
\text { factors }\end{array}$ & No & No & & {$[59-61,63,67,68]$} \\
\hline $\mathrm{TBM}^{\mathrm{a}}$ & $\begin{array}{l}\text { photon count } \\
\text { fluctuation }\end{array}$ & No & $?$ & $?$ & {$[53]$} \\
\hline $\mathrm{RBM}^{\mathrm{b}}$ & Geometric phase & No & Yes & No & {$[42]$} \\
\hline Microwave heating & Water absorption & Yes & No & Yes & [69] \\
\hline
\end{tabular}

${ }^{a}$ transnational Brownian motion;

${ }^{\mathrm{b}}$ rotational Brownian motion. 
is the most influencing factor that causes a frequency shift via the Zeeman effect and sin mixing. Given that the effect of the static magnetic field is canceled in the present method [Eqs. (3) and (4)], a slowly varying magnetic field, including the geomagnetic field, does not apparently induce artifacts if the magnetic field is static for more than $1.0 \mathrm{~s}$. The transverse magnetic field can affect the quantum thermometry via spin mixing because it can affect the ODMR shift, linewidth, and photon count. External stress to nanodiamonds is not likely to affect the ODMR measurements in most biological experiments because biological pressures are considerably small and do not affect the crystal field of NV centers in diamonds $(15 \mathrm{kHz} / \mathrm{MPa}[55,56])$. However, the strain inside nanodiamonds can affect the quantum thermometry via spin mixing, as discussed in this study. An electric field can also cause a frequency shift by inducing a stark shift $[57,58]$. While measuring local electric fields in a biological context has been proposed, such as a transmembrane electric field generated by the cell-membrane potential [2], a more critical situation would be when biomolecules possessing a large electron affinity are adsorbed on the ND surface, which is known to be adsorbed by various biomolecules in cells or living organisms [59-61]. Moreover, this mechanism has been used to manipulate the ND spin properties by surface functionalization $[62,63]$. Therefore, surface functionalization is important to control both the sensitivity and robustness of NV spin systems.

Other factors that are particular to biological environments include $\mathrm{pH}$, ionic strength, and water adsorption to the ND surface. Previous experiments based on the CW-ODMR spectral measurements have confirmed that these factors do not change both the ODMR frequency and the spectral shape in a wide range of $\mathrm{pH}$ and various ionic solutions [24,64]. As the precision of the CW-ODMR-based methods used in the literature was only in the range of $1-2^{\circ} \mathrm{C}$, a small effect within this precision range may be detected in the multipoint ODMR methods. It is also important to consider the effect of $\mathrm{pH}$ on the emission stability of negatively charged $\mathrm{NV}$ centers [70], because the emission instability causes variations in fluorescence intensity. In addition, NDs move randomly in cells and worms by the Brownian motion, intracellular transportation, and body motions [25,26,53]. These particle motions cause photon count fluctuations that may affect the multipoint ODMR measurements if the instrumental errors are not fully removed. Microwave irradiation may cause a change in the temperature during the measurement process due to microwave water heating that causes an ODMR shift [69]. Because microwaves are always irradiated during the measurements and would not affect the relative temperature measurements, local heating due to some conformational changes of biological samples may change the local dielectric permeability, thereby affecting the local heat-generation rate. Such artifacts also need to be considered if the observed temperature signal from the NV centers is complex.

One possible approach to validate the measured temperature data is comparing and/or combining the ODMR thermometry with all-optical NV thermometry [47,71-73] and other optical nanothermometry techniques [74-79]. These dual or multimodal temperature measurements are meaningful particularly for biosensing applications owing to the complex factors that may cause artifacts of the ODMR shift.

\section{CONCLUSION AND FUTURE PERSPECTIVES}

In this study, the experimental details and protocols regarding real-time multipoint ODMR measurements were presented (i.e., 3-point, 4-point, and 6-point methods). The multipoint ODMR measurement is a process used to estimate the frequency shift of the ODMR based on limited fluorescence intensity data at several frequency points. Therefore, a careful estimation of the frequency shift is required because unexpected factors may generate artifacts; the difference in the photo-responsivity between the photon counters is an example. In this study, the difference in photo-responsivity was shown to originate from the optical power dependence of the ODMR spectra. This optical power dependence is particularly prominent in the low photon regime and is only $0.5 \%$, which was not observed in the previous NV quantum experiments. When using multiple DAQ boards in the 6-point method, an instrumental error caused by the experimental hardware is further added. A practical method to cancel these artifacts is proposed, whereby the artifact values are subtracted from the obtained counter values based on the precharacterized photoresponsivity curves. Using developed real-time thermometry, the temperature of single NDs during a stepwise temperature change was measured successfully. Furthermore, the precision and noise sources were quantitatively compared of the 3-, 4-, and 6-point measurements. We also analyzed possible noise sources and artifacts in the quantum thermometry that should be considered in biosensing experiments.

A significant implication of this study is that quantum sensing requires both high sensitivity and effective implementation of sensors for realistic measurements. This study identifies a variety of artifact sources for real-time operation. The combination of multipoint ODMR measurements and particle tracking also requires significant care to avoid the artifacts. In this study, approximately half of the measurement time was used for particle tracking, and there is a potential to speed up the measurement process by implementing a simultaneous operation of particle tracking and temperature measurement. However, particle tracking is a feedback process used to maximize the fluorescence counts of NDs that move away from the focus and may be coupled with variations of fluorescence intensity derived from temperature changes in NV centers, particularly in the optically dynamic environment in the low-photon regime. The proposed particle tracking method is not significantly coupled with the temperature estimation because it is completely decoupled from the temperature estimation process; however, it may be coupled with thermometry when a fast particle-tracking algorithm is employed. Comparing or combining the ODMR thermometry with all-optical NV thermometry and other optical nanothermometry techniques is critical to confirm the results of the measured temperature data, particularly for biosensing applications owing to the complex factors that may cause artifacts of the ODMR shift. Studies regarding the implementation of quantum sensors into realistic measurement systems are crucial for the future development of quantum sensing.

\section{ACKNOWLEDGMENTS}

The authors thank J. Choi, H. Ishiwata, S. Inouye, E. Kage-Nakadai, N. Komatsu, M. D. Lukin, P. Maurer, 


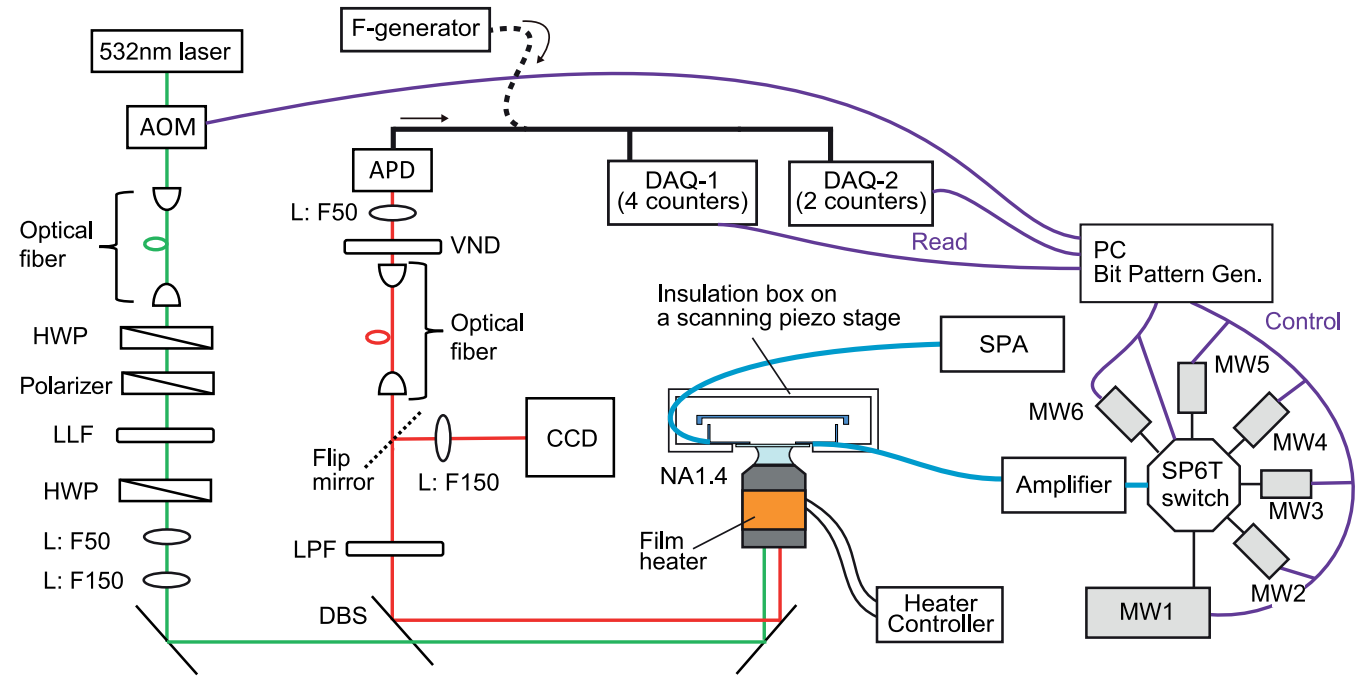

FIG. 10. Schematic drawing of the experimental setup for the optical layout and microwave circuit. VND: variable neutral density filter. LLF: laser-line filter. HWP: half-wave plate. L: lens. CCD: charge-coupled device camera. SPA: spectrum analyzer.

M. Turner, J. Twamley, Y. Umehara, R. Hira, R. Walsworth, and $\mathrm{H}$. Zhou for fruitful discussions and technical assistance in the experiments. The authors thank M. Roppongi at Institute for Center for Industry-University Innovation Support, Social Innovation and Cooperation, Utsunomiya University, for the measurement of a refractive index of immersion oils, Fig. 21. This study is partially supported by the Osaka City University Strategic Research Grant 2017 and 2018 (M.F., Y.S., A.D.) and JSPS-KAKENHI (M.F.: 16K13646, 17H02741, and 19K21935; Y.S.: 19K14636; M.F. and Y.S.: 20H00335). M.F. acknowledges funding by the MEXT-LEADER program, Sumitomo Research Foundation, and Murata Science Foundation. O.B. and A.D. acknowledge funding by the Deutsche Forschungsgemeinschaft DFG (FOR 1493 and SFB 951).

\section{APPENDIX A: EXPERIMENTAL APPARATUS}

Figure 10 shows the details of the confocal microscope and microwave excitation system. The following products were used for the main components: AOM: acousto-optic modulator (G\&H, 3200-121); DBS: dichroic beam splitter (Semrock, FF560-FDi01); LPF: long-pass filter (Semrock, BLP01-635R25); APD: avalanche photodiode (Excelitas, SPCM AQRH14); DAQ-1: data acquisition board (National Instruments, USB-6343 BNC); DAQ-2: data acquisition board (National Instruments, USB-6229 BNC); Optical fiber 1 (Thorlabs, 460-HP); Optical fiber 2 (Thorlabs, 1550HP); Piezo stage (Piezosystemjena, TRITOR-100SG); MW1: stand-alone microwave source (Rohde \& Schwarz, SMB100A); MW2-6: USB-powered microwave sources (Texio, USG-LF44); SP6T switch (General Microwave, F9160); Amplifier (Mini-circuit, ZHL-16W-43+); Bit Pattern gen.: bit pattern generator (Spincore, PBESR-PRO-300); F-generator (Stanford Research Systems, DS345). For the external temperature control, the following devices were used: Foil heater that wrapped the objective (Thorlabs, HT10K \& TC200, temperature precision: $\pm 0.1 \mathrm{~K}$ ); Immersion oil: Olympus Type-F; Pt100 resistance temperature probe (Netsushin, NFR-CF2-0505-30-100S-12000PFA-A-4); Handheld thermometer (WIKA, CTH7000);
Data logger for room temperature monitoring (T\&D, TR$72 \mathrm{wb})$.

\section{APPENDIX B: METHOD FOR THE MULTIPOINT SELECTION}

To determine the six frequency points at which the ODMR spectra are probed, the entire CW-ODMR spectral shape is considered for analysis. The analysis is divided into the following chronological steps:

1. The ODMR spectra are fitted using a sum of two Lorentzian functions in the form of

$$
\begin{aligned}
F_{\text {double }}(\omega)= & Y_{0}+2 A_{1}\left[\frac{\Gamma_{1}}{4\left(\omega-\bar{D}_{1}\right)^{2}+\Gamma_{1}^{2}}\right] \\
& +2 A_{2}\left[\frac{\Gamma_{2}}{4\left(\omega-\bar{D}_{2}\right)^{2}+\Gamma_{2}^{2}}\right]
\end{aligned}
$$

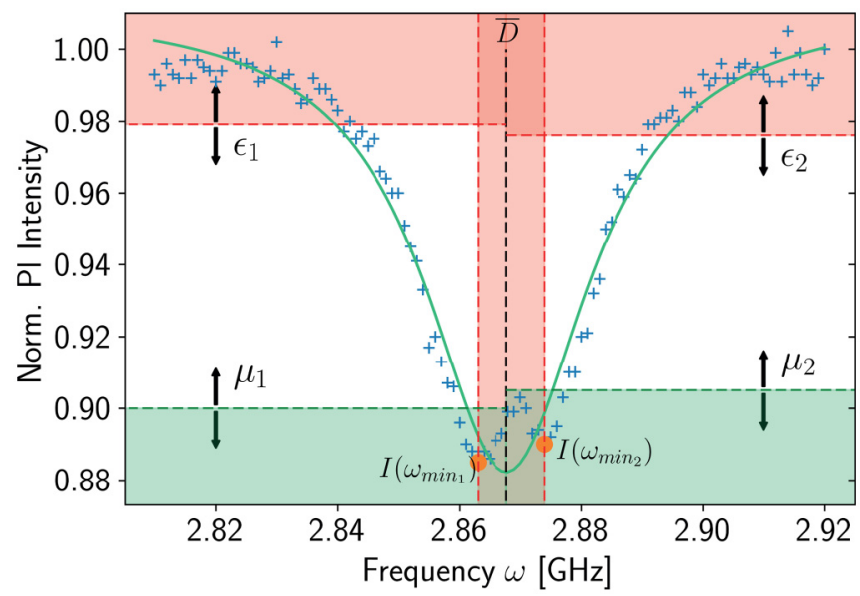

FIG. 11. ODMR spectrum showing areas of data points that have been excluded prior to the fitting of the slopes (red areas) for either being part of the baseline noise (regulated via $\epsilon_{1,2}$ ) or dip noise (i.e., being in between the two local minima), and post the fitting (green area) for being in a considerably close proximity to the dip (regulated via $\mu_{1,2}$ ). A single Lorentzian dip (green line) indicates the approximate position of the zero-field splitting (dotted black line). 
TABLE III. Variation of $\gamma_{1}$ and $\gamma_{2}$ in the 4-point selection process.

\begin{tabular}{lccc}
\hline \hline Sample ND & $\gamma_{1}\left[\mathrm{MHz}^{-1}\right]$ & $\gamma_{2}\left[\mathrm{MHz}^{-1}\right]$ & Difference [\%] \\
\hline 1 & -4.821 & 4.594 & 4.7 \\
2 & -8.807 & 9.112 & 3.3 \\
3 & -6.152 & 5.825 & 5.6 \\
4 & -9.326 & 8.825 & 5.4 \\
5 & -4.440 & 4.194 & 5.9 \\
\hline \hline
\end{tabular}

to account for the increased dip splitting. Here, $Y_{0}, A_{i}, \Gamma_{i}$, $\bar{D}_{i}$ denote the global offset, spectral area, HWHM, and dip frequency of the $i$ th Lorentzian, respectively. However, this double Lorentzian was simplified using a single Lorentzian as follows:

$$
F_{\text {single }}(\omega)=Y_{0}+2 A_{1}\left[\frac{\Gamma}{4(\omega-D)^{2}+\Gamma^{2}}\right] .
$$

The zero-field splitting $(D)$ is then approximately given by

$$
\bar{D} \approx D=\bar{D}_{1}+\frac{\overline{D_{2}}-\bar{D}_{1}}{2} \text {. }
$$

This approximation is valid because the difference between $D$ and $\bar{D}$ is one or two orders of magnitude smaller than the linewidth.

2. Each domain is then checked for a local intensity minimum and data points to the right of the local minimum $(\omega>$ $\left.\omega_{\min 1}\right)$ in the first domain. Data points to the left of the local minimum $\left(\omega>\omega_{\min 1}\right)$ in the second domain are extracted, as indicated in Fig. 11. This is an important step in case of dip splitting as it sets the ground for a solid first guess for the linear fit for the two slopes.

3. Data points that can be regarded as baseline noise are specified on each slope. This is done by comparing the absolute ODMR dip intensity value of that data point, i.e., $\left|I_{\text {dip }}(\omega)\right|=\left|I(\omega)-Y_{0}\right|$ to the proposed preset fraction of the maximum value of the ODMR dip intensity (i.e., $\left|I_{\text {dip }}^{\text {base }}\left(\omega_{\min 1,2}\right)\right|=\epsilon_{1,2}\left|I\left(\omega_{\min 1,2}\right)-Y_{0}\right|$ with $\left.0 \leqslant \epsilon_{1,2} \leqslant 1\right)$. In the case that $\left|I_{\text {dip }}(\omega)\right|<I_{\text {dip }}^{\text {base }}\left(\omega_{\min 1,2}\right) \mid$, the data point is excluded (Fig. 11).

4. The two linear slopes of the ODMR spectrum are determined by linear fits. In the first step of each iteration, the routine generates two data sets, of which the first misses the first element and the second misses the last element of the initial data set. Both datasets are then fitted, and the resulting residues are compared. The data set with the lower residue is passed on. Therefore, the extent of the linear slope is narrowed down. The routine is executed until a preset maximum-allowed residue is surpassed, or the number of elements in the data set reaches the minimum-allowed amount.

5. Two functions are formulated based on the fitting results of the two linear slopes. One can also exclude the dip noise on each slope (i.e., parts of the linear function that are in close approximation to the dip and, hence, likely to become noisy during experiments with high temperature changes). This is done similarly as step 3 by excluding those parts for which $\left|I_{\text {Fit }}(\omega)\right|>\left|I_{\text {dip }}^{\text {ref }}\left(\omega_{\min 1,2}\right)\right|$ with $\left|I_{\text {dip }}^{\text {ref }}\left(\omega_{\min 1,2}\right)\right|=$ $\mu_{1,2}\left|I\left(\omega_{\min 1,2}\right)-Y_{0}\right|$ and $0 \leqslant \mu_{1,2} \leqslant 1$.

6. To match the requirement for Eq. (3), $I\left(\omega_{-}\right)=I\left(\omega_{+}\right)$ pairs are allocated to one another and the two frequencies $\omega_{-}$ and $\omega_{+}$of the pair that exhibit the highest collective $\delta \omega$ within the extent of the slopes are selected as $\omega_{-}$and $\omega_{+}$.

7. The four frequency points, two on each slope, are uniformly distributed (i.e., equally distanced with $\delta \omega$ from $\omega_{-}$ and $\left.\omega_{+}\right)$, as depicted in Fig. 11.

\section{APPENDIX C: DERIVATION OF THE FLUORESCENCE INTENSITY AT FOUR FREQUENCY POINTS}

The ODMR intensities $I(\omega)$ at the four frequency points can be written as

$$
\begin{aligned}
& I_{1}=I\left(\omega_{-}\right)+\gamma_{1}\left[-\delta \omega+\delta \beta+\delta T\left(\frac{d D}{d T}\right)\right], \\
& I_{2}=I\left(\omega_{-}\right)+\gamma_{1}\left[+\delta \omega+\delta \beta+\delta T\left(\frac{d D}{d T}\right)\right], \\
& I_{3}=I\left(\omega_{+}\right)+\gamma_{2}\left[-\delta \omega-\delta \beta+\delta T\left(\frac{d D}{d T}\right)\right], \\
& I_{4}=I\left(\omega_{+}\right)+\gamma_{2}\left[+\delta \omega-\delta \beta+\delta T\left(\frac{d D}{d T}\right)\right],
\end{aligned}
$$

where $\gamma_{1}$ and $\gamma_{2}$ depict the slopes of the two linear domains and $d D / d T=\alpha$ indicates the temperature dependence of $D$. (a)

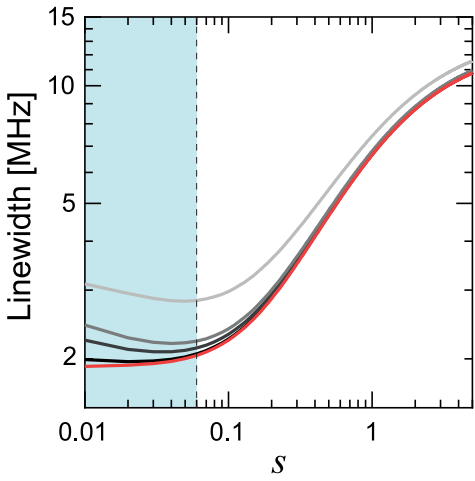

(b)

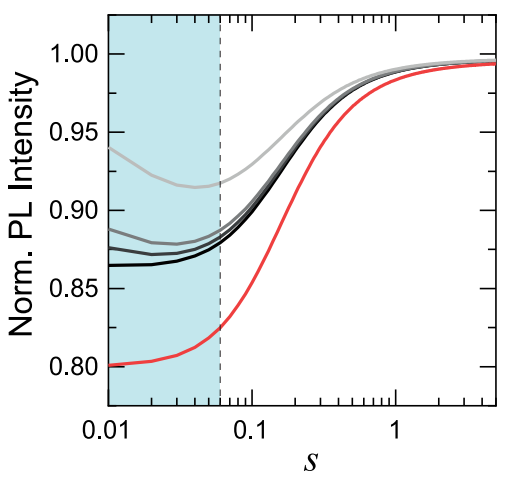

$-\Gamma_{2}^{*}=0.1[\mathrm{MHz}], \Gamma_{1}=1 \quad[\mathrm{kHz}]$

$-\Gamma_{2}^{*}=0.5[\mathrm{MHz}], \Gamma_{1}=5 \quad[\mathrm{kHz}]$

$-\Gamma_{2}^{*}=1.0[\mathrm{MHz}], \Gamma_{1}=10[\mathrm{kHz}]$

$-\Gamma_{2}^{*}=5.0[\mathrm{MHz}], \Gamma_{1}=50[\mathrm{kHz}]$

High intensity approximation

FIG. 12. Simulated optical power dependence of the linewidth (a) and contrast (b) of ODMR spectra for different sets of spin relaxation times $\left(\Gamma_{2}^{*}, \Gamma_{1}\right)$. The red line indicates the approximation for the high optical intensity that does not present the influences of the spin relaxation times. 
(a)

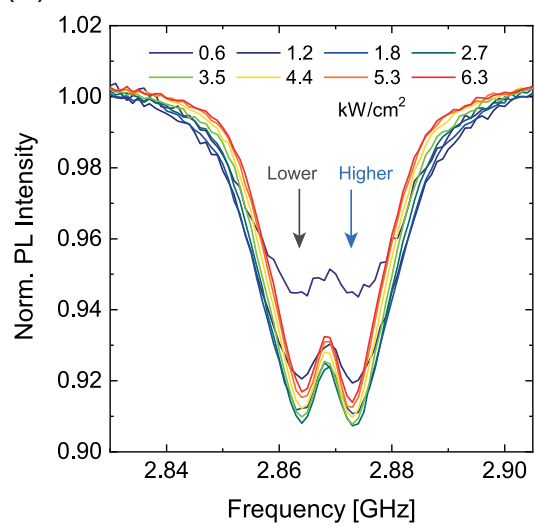

(d)

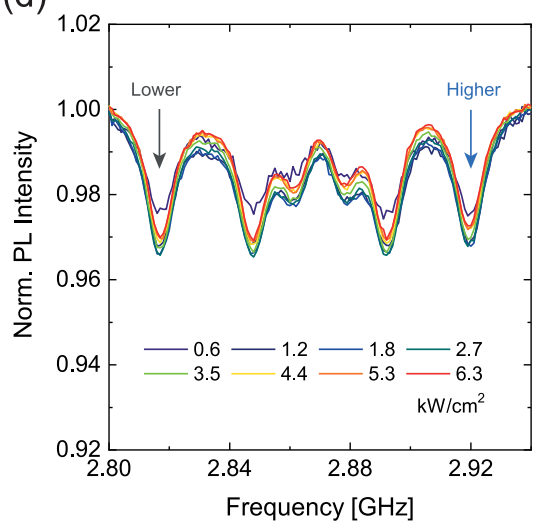

(b)

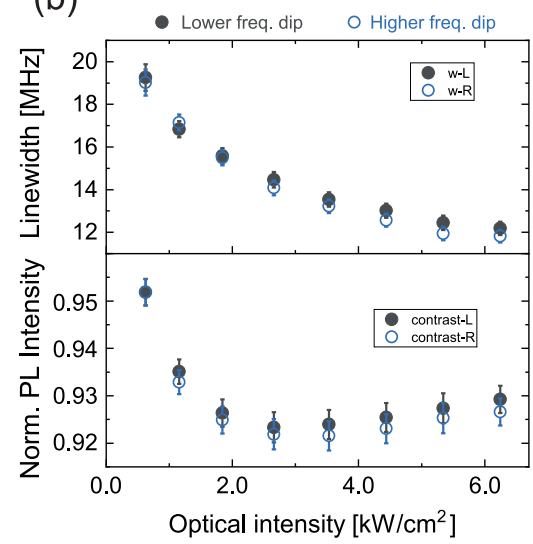

(e)

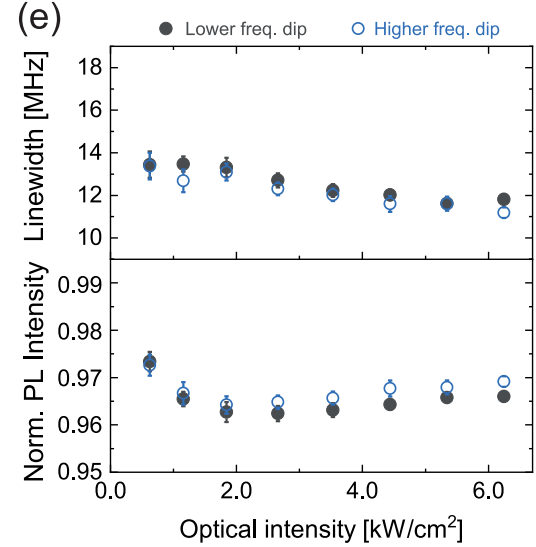

(c)

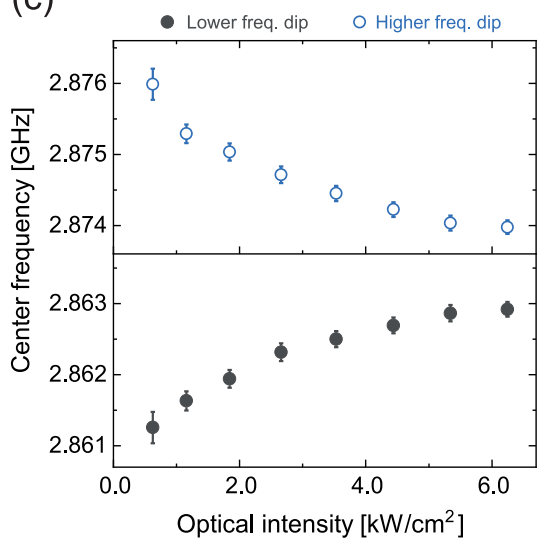

(f)

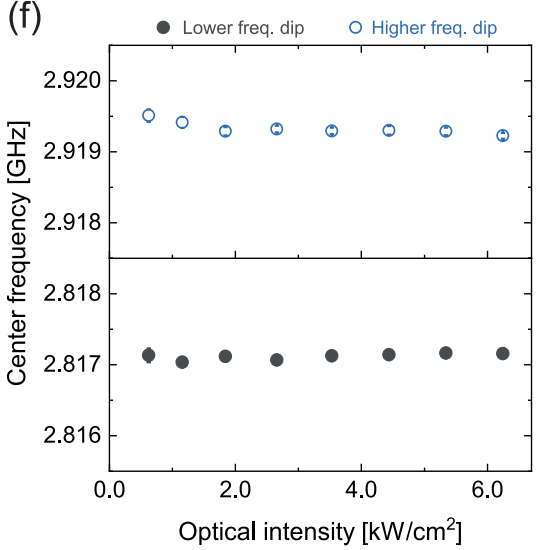

FIG. 13. ODMR spectra for different optical excitation intensities under zero magnetic field (0 G) (a) and the external magnetic field (32 G) (d). The optical power dependence of the linewidth (b), (e) and contrast (e), (f) for $0 \mathrm{G}$ and $32 \mathrm{G}$, respectively. The shaded area indicates the present measurement region of $s<0.06$.

$\delta \beta$ is an unknown static magnetic field [21], but is assumed to be zero in this investigation. $\left|\gamma_{1}\right|$ and $\left|\gamma_{2}\right|$ are assumed to be equal; however, they exhibit slight differences $(\sim 50 \%)$, as shown in Table III.

\section{APPENDIX D: INFLUENCE OF OPTICAL POWER AND WEAK MAGNETIC FIELD TO THE ODMR SPECTRA}

The optical power dependence of the ODMR spectral shape was theoretically analyzed in Ref. [41] by introduc- (a)

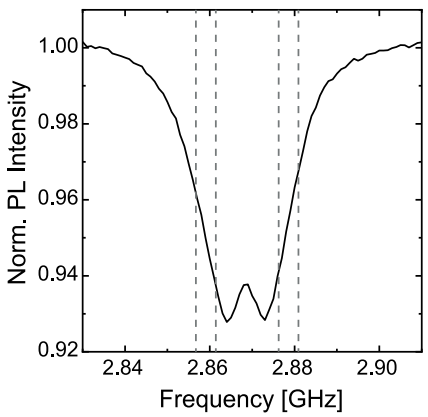

(b)

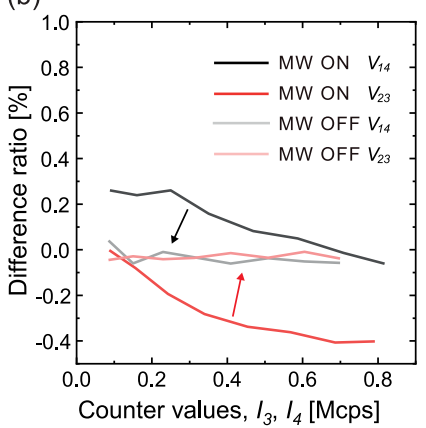

FIG. 14. Effect of the nonlinearity in the photo-responsivity of APDs to $V_{i j}$. (a) CW-ODMR spectrum with four selected frequencies. (b) Comparison between the microwave-ON of $V_{14}, V_{23}$, and their microwave-OFF profiles in the 4-point measurement. ing the optical decoherence and optical saturation effect to the Bloch equation formalism, which provides the following equations for the linewidth $(\Delta v)$ and ODMR contrast $(C)$ :

$$
\begin{aligned}
\Delta v & =\frac{1}{2 \pi} \sqrt{\Gamma_{2}^{2}+\frac{\Omega_{R}^{2} \Gamma_{2}}{2 \Gamma_{1}+\Gamma_{p}}}, \\
C & =\frac{1}{2} \frac{(\alpha-\beta) \Gamma_{p}}{(\alpha+\beta) \Gamma_{1}+\alpha \Gamma_{p}} \frac{\Omega_{R}^{2}}{\Omega_{R}^{2}+\Gamma_{2}\left(2 \Gamma_{1}+\Gamma_{p}\right)},
\end{aligned}
$$

where $\Gamma_{2}=\Gamma_{2}^{*}+\Gamma_{c}^{\infty} s(1+s)^{-1}$ and $\Gamma_{p}=\Gamma_{p}^{\infty} s(1+s)^{-1}$. Here, $\Omega_{R}$ is Rabi frequency, and $\Gamma_{1}, \Gamma_{2}^{*}, \Gamma_{c}^{\infty}$, and $\Gamma_{p}^{\infty}$ are the spin-lattice relaxation rate, inhomogeneous dephasing rate, polarization rate at an optical saturation that is fixed by the lifetime of the metastable state, and the rate of optical cycles at the saturation set by the excited-state lifetime, respectively. $s$ is the optical saturation parameter defined as $s=I / I_{S}$, where $I_{S}$ is the saturation intensity. Considering the ODMR contrast, $\alpha$ and $\beta$ are the phenomenological parameters accounting for the difference in the spin population of the $m_{S}=0$ and $m_{S}=$ 1 spin sublevels. Using these equations, the optical power dependence of $\Delta v$ and $C$ is simulated for different sets of spin relaxation rates, as shown in Fig. 12. Specifically, the same parameters used in Ref. [41] were utilized for $\Omega_{R}=$ $3 \times 10^{6} \mathrm{rad} \mathrm{s}^{-1}, \Gamma_{c}^{\infty}=80 \mathrm{MHz}$, and $\Gamma_{p}^{\infty}=5 \mathrm{MHz} ; \Gamma_{2}^{*}, \Gamma_{1}$ were varied to analyze how the optical power dependency is affected by these spin relaxation parameters. The linewidth 
(a)

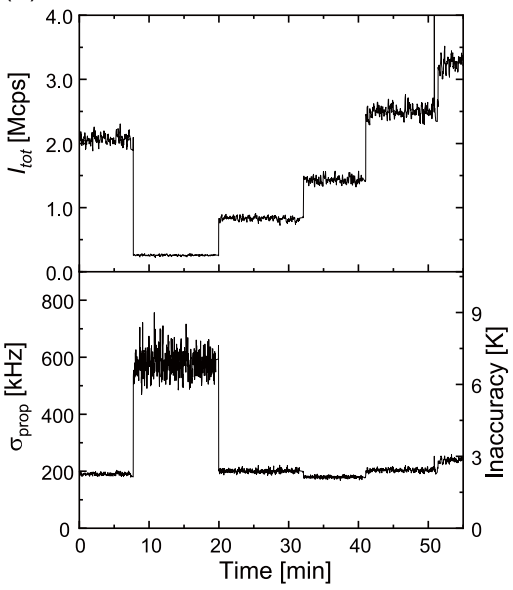

(b)

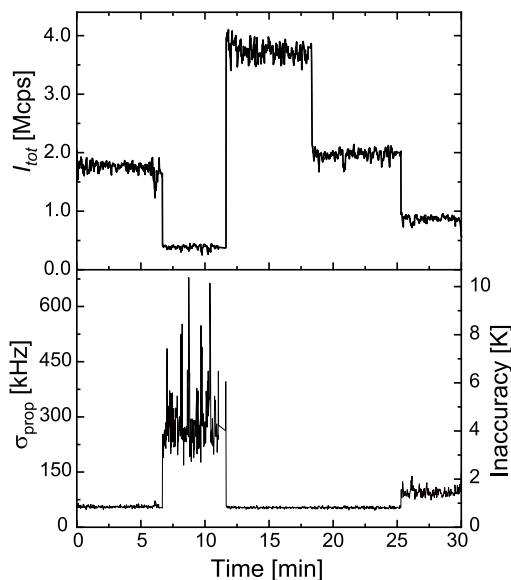

(c)

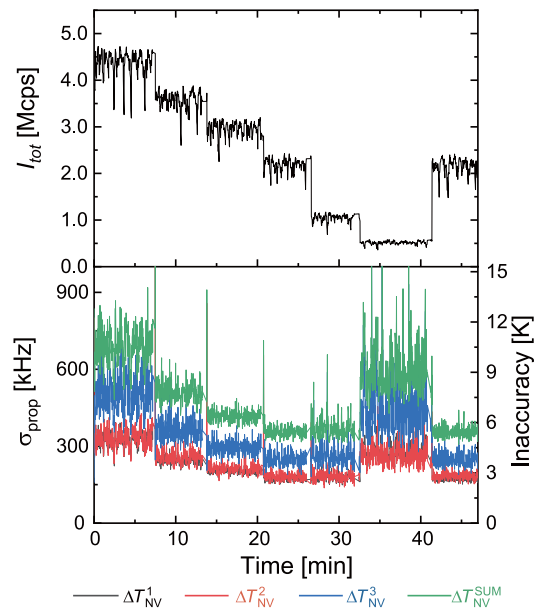

FIG. 15. Error propagation to the temperature estimation of $\Delta T_{\mathrm{NV}}$. $I_{\mathrm{tot}}$ (top) and propagated errors, $\sigma_{\text {prop }}$ (bottom) for the (a) 3-point, (b) 4-point, and (c) 6-point measurements.

and ODMR contrast are significantly dependent on the spin relaxation parameters in the low photon regime of $s<0.1$. In contrast, this dependency vanishes when approximating the high photon regime as $\Gamma_{p} \gg \Gamma_{1}$ and $\Gamma_{2} \sim \Gamma_{c}^{\infty} s(1+s)^{-1}$. These simulations support the optical power dependence of the ODMR spectra (Fig. 3) in the present observation. Note that the data were first aimed to be fitted with these equations; however, it was difficult to obtain reliable fitting results owing to the excessive number of relaxation parameters. Furthermore, the level of anticrossing was observed to significantly affect the relaxation parameters, as indicated below; the resultant fitting parameters were not verified. Therefore, considering this computation, we only understand that the principal trend of the linewidth and contrast can be expressed by the Bloch equation incorporating the optical effects.

While the aforementioned formulations based on the Bloch equations are capable of indicating the optical power dependence of the ODMR spectra observed in this study, it does
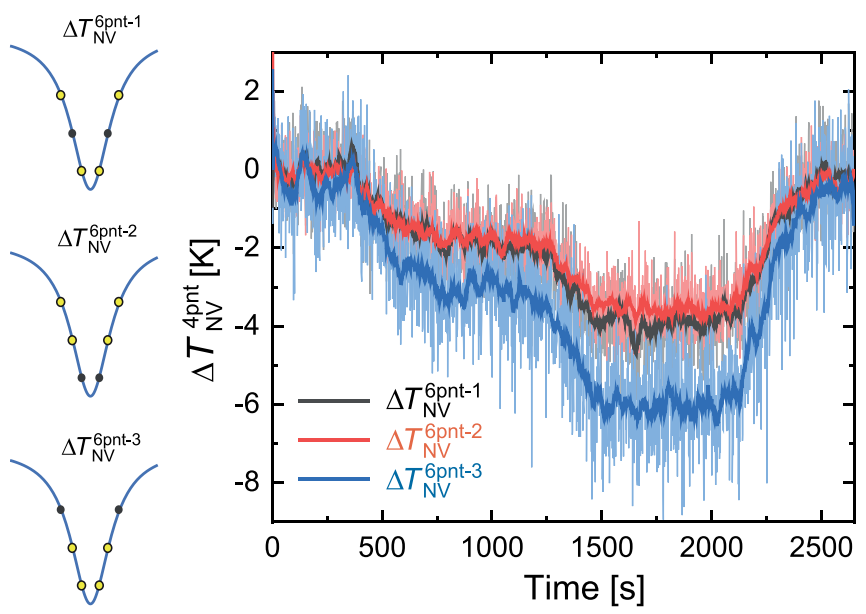

FIG. 16. A schematic illustration of the chosen points for the 6-point method and the resultant time profiles of $\Delta_{\mathrm{NV}}^{6 \mathrm{pnt}}$ during the stepwise temperature change shown in Fig. 8. not address the mechanism of the center frequency shift. To determine the center frequency shift, the influence of the external magnetic field on the optical power dependence is analyzed. Figure 13 presents the power dependence of the ODMR spectra of the same ND without a magnetic field (0 $\mathrm{G})$, and with an external magnetic field (32 G). Here, the magnetic field was applied by approaching a small magnet (the magnetic field vector was basically aligned to the NV axis such that the splitting of the ODMR spectra was maximum). The total data acquisition times were $15 \mathrm{~h}$ and $19 \mathrm{~h}$ for the 0 $\mathrm{G}$ and $32 \mathrm{G}$ magnetic fields, respectively. The results show that the frequency shifts of the ODMR dips for $m_{\mathrm{S}}= \pm 1$ nearly disappeared (variation reduced from $2 \mathrm{MHz}$ at $0 \mathrm{G}$ to $0.2 \mathrm{MHz}$ at $32 \mathrm{G}$ ). In addition, the optical power dependence of the linewidth and ODMR contrast are significantly reduced, indicating that the spin relaxation times are changed by the external magnetic field.

\section{APPENDIX E: EFFECT OF NONLINEARITY IN THE PHOTO-RESPONSIVITY OF APD TO $V_{i j}$ IN THE 4-POINT MEASUREMENT}

APD has a nonlinearity in the photo-responsivity, which may affect the $V_{i j}$ variation. Figure 14 compares the variation of $V_{i j}$ between the microwave excitation when it was $\mathrm{ON}$ and OFF because $V_{i j}$ when the microwave is OFF should reflect the effect of APD nonlinearity.

\section{APPENDIX F: ERROR PROPAGATION OF THE SECOND-ORDER POLYNOMIAL FIT TO THE TEMPERATURE ESTIMATION}

The errors of the fitting parameters summarized in Table I propagate to the temperature estimation using Eqs. (3)-(5), which can be explicitly expressed as Eqs. (F2)-(F3). Figure 15 presents the propagated errors associated with the time trace in Fig. 7(a): 


$$
\begin{aligned}
\sigma_{\text {prop }}^{3 \mathrm{pnt}}= & -\Gamma \frac{1+\rho^{2}}{2 \rho} \sqrt{\frac{4\left(I_{0}-I_{-}\right)^{2}\left[\delta c_{0}^{2}+\left(I_{+}^{\mathrm{NC}}\right)^{2}\left[\delta c_{1}^{2}+\left(I_{+}^{\mathrm{NC}}\right)^{2} \delta c_{2}^{2}\right]\right.}{\left(2 I_{0}-I_{-}-I_{+}^{\mathrm{C}}\right)^{4}}}, \\
\sigma_{\text {prop }}^{4 \mathrm{pnt}}= & \frac{2 \delta \omega}{\left[I_{1}^{C}-I_{2}^{C}-\left(I_{3}^{C}-I_{4}^{C}\right)\right]^{2}} \\
& \times \sqrt{\left(I_{4}^{C}-I_{2}^{C}\right)^{2}\left\{\delta a_{0}^{2}+\left(I_{3}^{\mathrm{NC}}\right)^{2}\left[\delta a_{1}^{2}+\left(I_{3}^{\mathrm{NC}}\right)^{2} \delta a_{2}^{2}\right]\right\}+\left(I_{1}^{C}-I_{3}^{C}\right)^{2}\left\{\delta b_{0}^{2}+\left(I_{4}^{N C}\right)^{2}\left[\delta b_{1}^{2}+\left(I_{4}^{N C}\right)^{2} \delta b_{2}^{2}\right]\right\}}, \\
\sigma_{\text {prop }}^{6 \mathrm{pnt}}= & \sqrt{\left[\sigma_{\text {prop }}^{4 \mathrm{pnt}}\left(I_{1}, I_{2}, I_{3}, I_{4}\right)\right]^{2}+\left[\sigma_{\mathrm{prop}}^{4 \mathrm{pnt}}\left(I_{1}, I_{-}, I_{+}, I_{4}\right)\right]^{2}+\left[\sigma_{\text {prop }}^{4 \mathrm{pnt}}\left(I_{-}, I_{2}, I_{3}, I_{+}\right)\right]^{2}} .
\end{aligned}
$$

\section{APPENDIX G: THREE TYPES OF STEPWISE TEMPERATURE PROFILES IN THE 6-POINT MEASUREMENT}

Figure 16 shows the time profiles of each 4-point measurement conducted in the 6-point measurements. $\Delta_{\mathrm{NV}}^{6 \mathrm{pnt}-3}$ that probes the bottom region overestimates the temperature change by a factor of 1.7 compared to the other estimates, which indicates that the bottom region is more shifted than the base region of the ODMR dip.

\section{APPENDIX H: EFFECT OF THE SPECTRAL SHAPE TO THE TEMPERATURE ESTIMATION IN THE 3-POINT METHOD}

In the 3-point method, the real spectral shape of the ODMR does not match the single Lorentzian profile, which causes deviations in the estimation from the real temperature change. Figure 17 shows how the spectral shape affects the temperature estimates in the 3-point method for the ND used in Fig. 8. First, the spectral functions are formulated by fitting the experimentally measured ODMR spectrum [Fig. 17(a)] with a single Lorentzian, Gaussian, and pseudo-Voigt function. In addition, an interpolated function of the experimental spectrum is formulated. Second, these four spectral functions are shifted assuming the temperature change, and $\Delta T$ is numerically estimated by the 3-point method [Eq. (2)]. When the spectral shape is a perfect single Lorentzian, the 3-point method accurately estimates the temperature change, as shown in Fig. 17(b). However, the 3-point method exhibits a significant deviation from the Lorentzian-based estimation when the spectral shape is Gaussian or pseudo-Voigt [Figs. 17(c) and 17(d)]. The deviation increases as the frequencies of $I_{-}$and $I_{+}$increase. In case of the real ODMR spectrum (interpolated function), the deviation is significant.

The off-resonant photon count $\left(I_{0}\right)$ also affects temperature estimation. Figures 18(a) and 18(b) show the time profiles of the stepwise temperature change measured by the 3-point method when the microwave irradiation at $2.65 \mathrm{GHz}$ is $\mathrm{ON}$ or OFF during the measurement of $I_{0}$, respectively. The irradiation of the far off-resonant $2.65 \mathrm{GHz}$ should provide the same photon count as when no microwave is irradiated; however, $I_{0}$ is slightly increased $(\sim 1 \%)$ when the $2.65-\mathrm{GHz}$ microwave is irradiated in the present experiment, resulting in the increase of the temperature dependency of the zero-field splitting $(\alpha)$ from -95 to $-105 \mathrm{kHz} / \mathrm{K}$. This change of $I_{0}$ by the microwave irradiation is most likely caused by the high-frequency noise exerting on to the piezo stage or other electronic devices because a significantly small level of noise effect to the electronics of the piezo stage were detected (e.g., the microwave irradiation seems causing the positional shift of laser focal point on the order of $10 \mathrm{~nm}$ ). While it may be

(a)

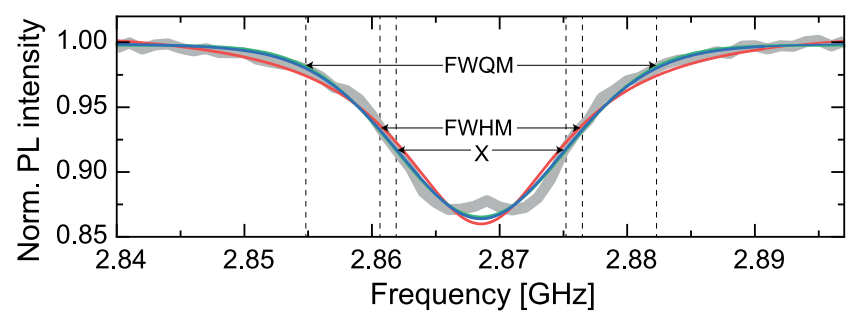

(b)

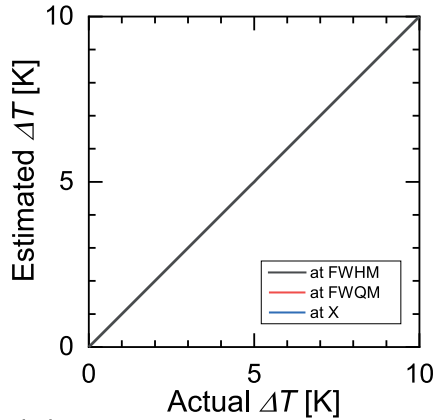

(c) (d)

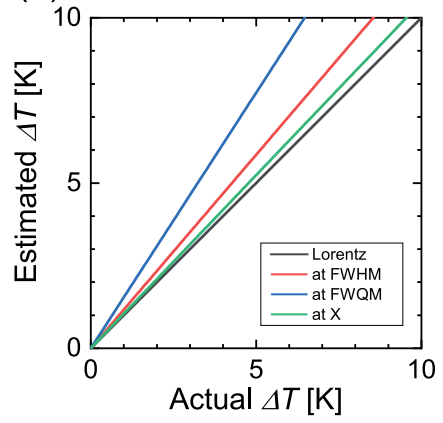

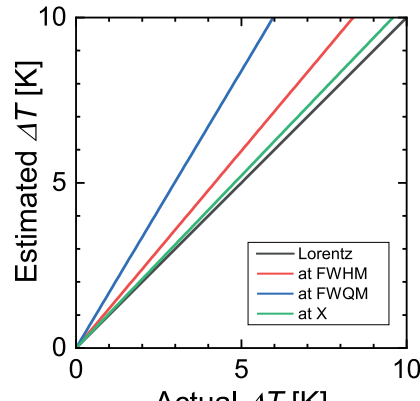

Actual $\Delta T[\mathrm{~K}]$

(e)

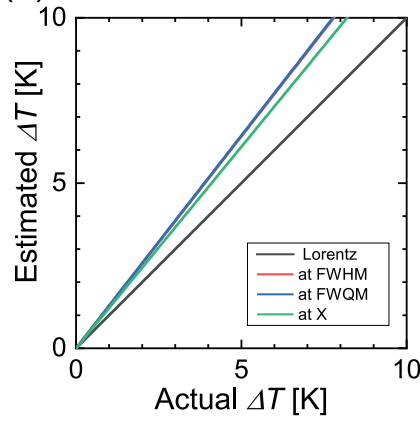

FIG. 17. (a) The ODMR spectrum used in Fig. 8 is fitted by a single Lorentzian (red), Gaussian (blue), and pseudo-Voght (green) function. The theoretical analysis regarding the relationship between the estimated $\Delta T$ and actual $\Delta T$, when the ODMR spectrum is considered as (b) Lorentzian, (c) Gaussian, (d) pseudo-Voght, and (e) interpolating function, respectively. 
(a)

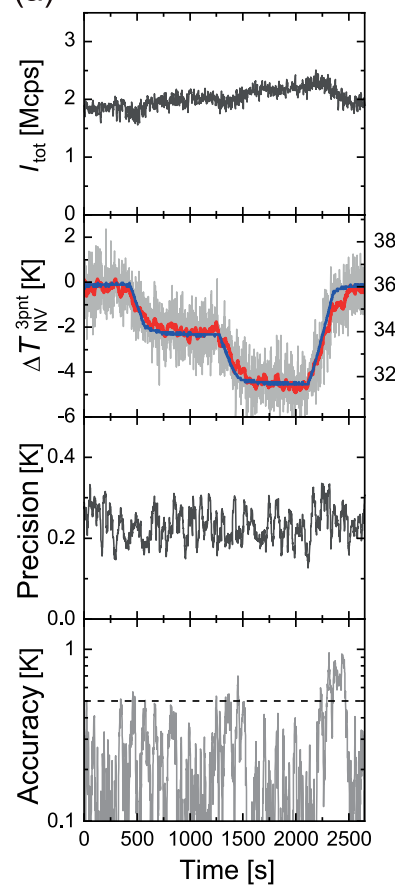

(b)

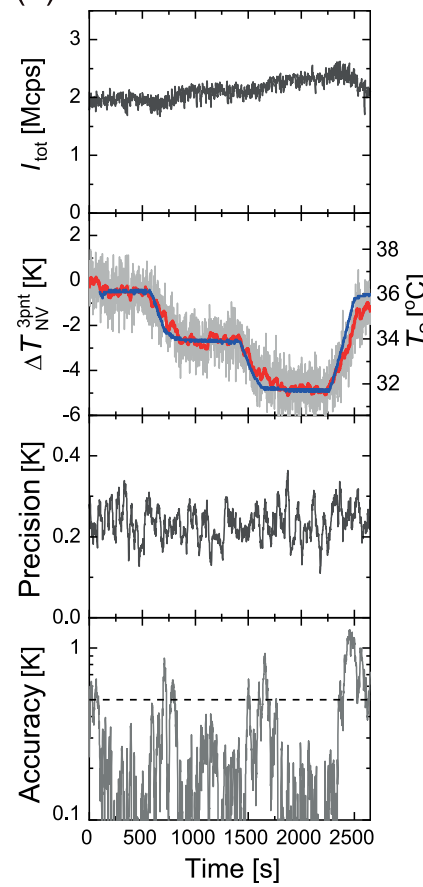

(c)

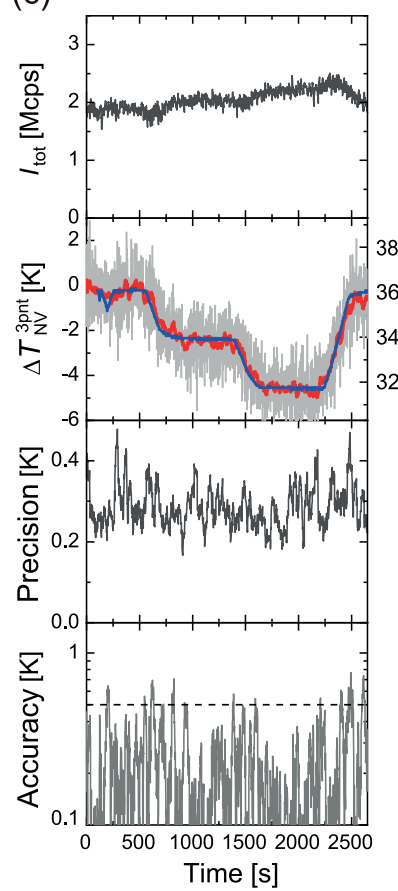

(d)

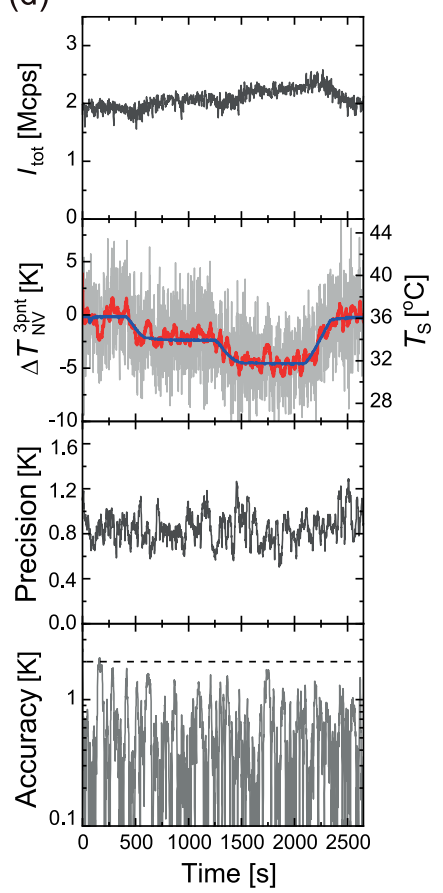

FIG. 18. Time profiles of $\Delta T_{\mathrm{NV}}$ for the 3-point methods by different experimental parameters. The time profile in (a) the normal configuration [the same as in Fig. 8(b)] and (b) when the microwave at $2.65 \mathrm{GHz}$ is switched off during the measurement of $I_{0}$. (c) The time profiles when the frequencies of $I_{-}$and $I_{+}$are set to FWHM and (d) FWQM (full-width at quarter maximum) (d).

possible to completely remove the high-frequency noise, the observed sensitivity of the 3-point method to the variation of $I_{0}$ may affect the measurement when working on biological samples because water has an absorption at $2.65 \mathrm{GHz}$. The observed sensitivity of the 3-point method fundamentally comes from the fact that the $I_{0}$ term is not completely canceled in

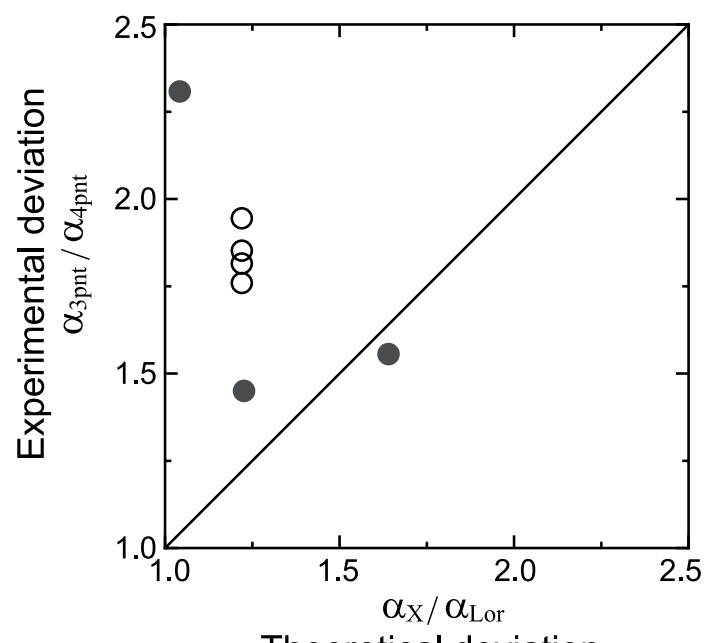

Theoretical deviation

FIG. 19. Experimental deviation of $\alpha_{3 \text { pnt }} / \alpha_{4 \text { pnt }}$ against the theoretical deviation estimated from the spectral shape of the ODMR $\left(\alpha_{\mathrm{X}} / \alpha_{\text {Lor }}\right)$. The line with a slope of 1 is a visual guide. The open circles are of the same ND, but are measured in different measurement parameters, as shown in Fig. 18.

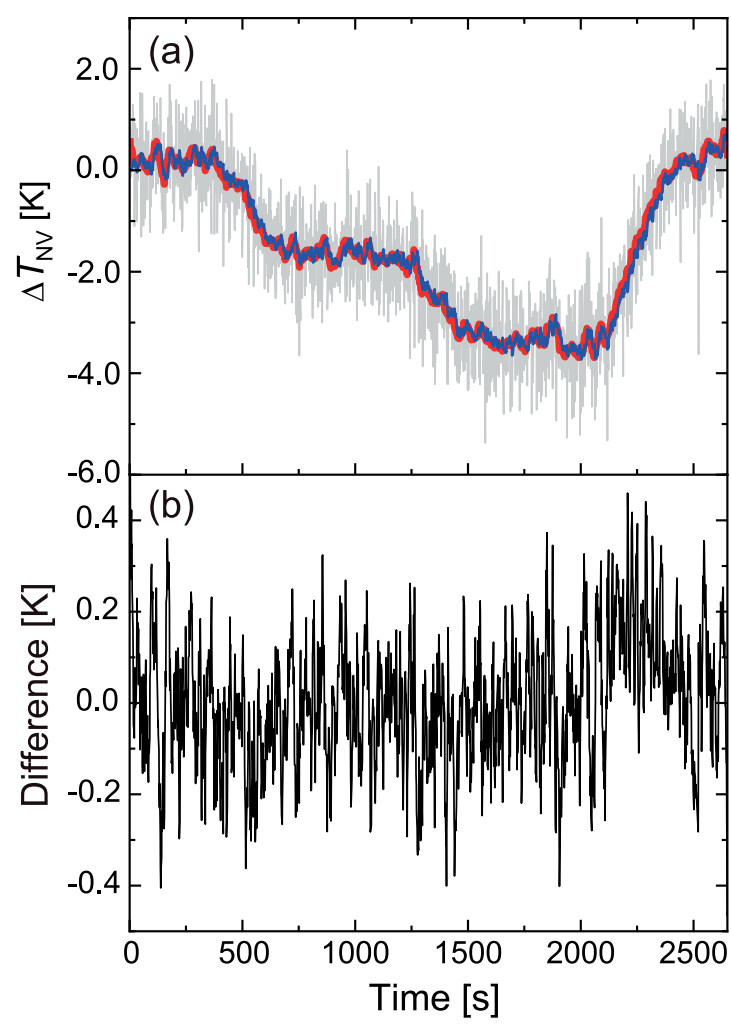

FIG. 20. (a) Time profile of $\Delta T_{\mathrm{NV}}^{4 \mathrm{pnt}}$ (gray) with 20-point moving average (red) and Kalman filter (blue). For Kalman filtering, $\sigma_{p}^{2}=0.01$ and $\sigma_{m}^{2}=1$ were used. (b) Difference between the moving average and Kalman filtering. 


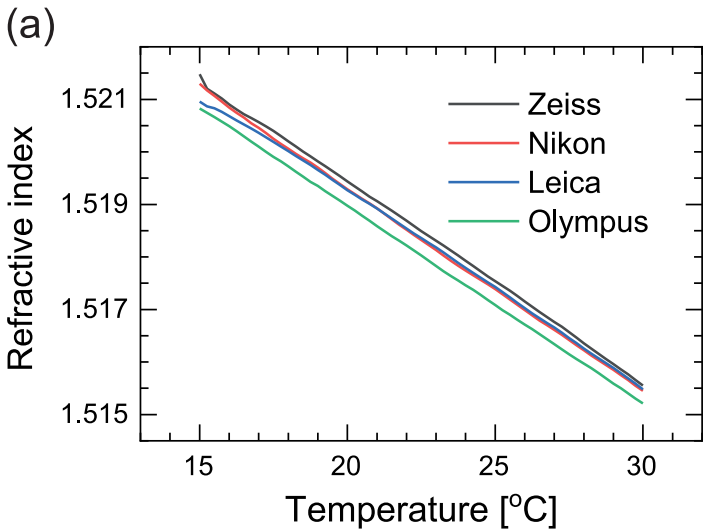

(b)

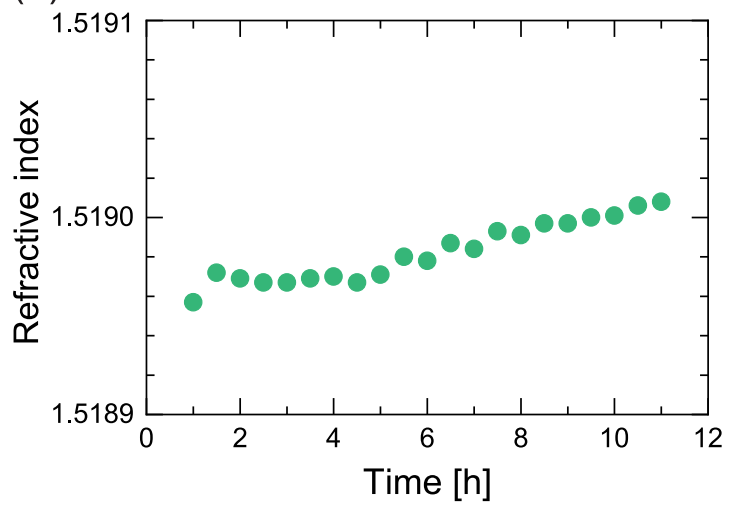

FIG. 21. (a) Refractive index of immersion oils from Zeiss, Nikon, Leica, and Olympus as a function of temperature from $15^{\circ} \mathrm{C}$ to $30^{\circ} \mathrm{C}$ by $0.25^{\circ} \mathrm{C}$. (b) Long-term variation of the refractive index from Olympus at $20^{\circ} \mathrm{C}$.

Eq. (2) in contrast to the formulation of Eq. (3). In addition to the sensitivity to $I_{0}$, the selection of the two frequency points on the slope of the ODMR dip affects the estimation, as shown in Figs. 18(c) and 18(d), where the two frequencies were set to the FWHM and FWQM points. In particular, the measurement at the FWQM points is noisy owing to the small contrast of the ODMR between the off-resonance and measurement points.

For the four NDs, we plotted the ratio of $\alpha$ between the 4- and 3-point methods against the theoretical deviation that can be expected from the spectral shape by the temperature shifting of the interpolated function, as shown in Fig. 19. The experimental deviation is usually larger than the theoretical deviation. This trend suggests that there should be other causes to the present excessive estimation of $\alpha$ of the 3-point method. A more thorough analysis will be necessary in the future development of the 3-point method.

TABLE IV. Fitting parameters for the temperature dependence of the refractive index.

\begin{tabular}{lcc}
\hline \hline Company & Intercept & Slope $\left[\times 10^{-4}{ }^{\circ} \mathrm{C}^{-1}\right]$ \\
\hline Zeiss & 1.52709 & -3.82786 \\
Nikon & 1.52701 & -3.85555 \\
Leica & 1.52665 & -3.70161 \\
Olympus & 1.52648 & -3.75796 \\
\hline \hline
\end{tabular}
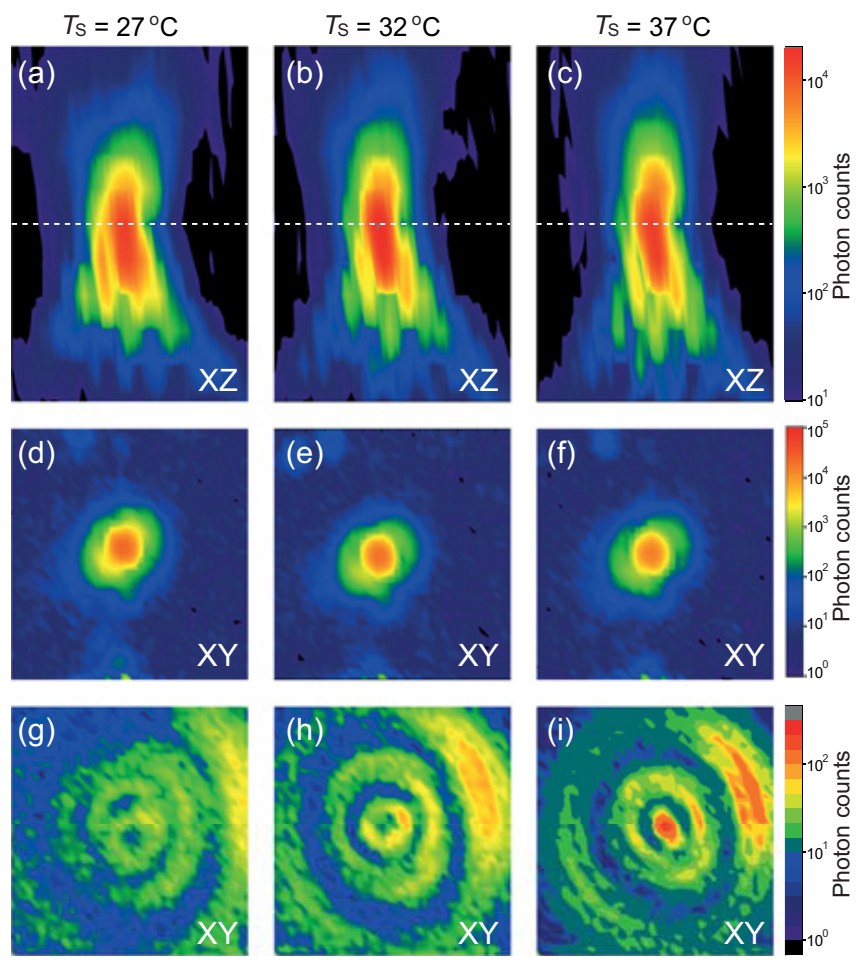

FIG. 22. Visualized PSFs in the present experiments at different temperatures with log-scale coloring. $X Z$ planes at $T_{\mathrm{S}}=27^{\circ} \mathrm{C}$ (a), $32^{\circ} \mathrm{C}$ (b) and $37^{\circ} \mathrm{C}(\mathrm{c})$. The vertical side and horizontal side are $4.8 \mu \mathrm{m}$ and $3.2 \mu \mathrm{m}$, respectively. The corresponding $X Y$ planes at $Z=0 \mu \mathrm{m}$ and $Z=-0.3 \mu \mathrm{m}$ at $T_{\mathrm{S}}=27^{\circ} \mathrm{C}(\mathrm{d}),(\mathrm{g}), 32^{\circ} \mathrm{C}(\mathrm{e}),(\mathrm{h})$ and $37^{\circ} \mathrm{C}$ (f), (i). The both sides are $3.2 \mu \mathrm{m}$. In the $X Z$ plane, the dashed lines indicate $Z=0 \mu \mathrm{m}$ at which the interface of glass (lower region) and air (upper region) exists. Photon counts are per $10 \mathrm{~ms}$.

\section{APPENDIX I: NOISE FILTERING FOR CORRECT TEMPERATURE ESTIMATION}

The multipoint ODMR measurements are essentially an estimation of the temperature-dependent frequency shift of ODMR, which motivates the implementation of other noise filters and data estimation techniques for the transient data set. A suitable choice is the Kalman filter, which has been widely used in systems and control engineering [80]. The following (one-dimensional) Kalman filter was employed:

$$
\begin{aligned}
\hat{x}_{k+1} & =\hat{x}_{k}^{\prime}+G\left(z_{k}-\hat{x}_{k}^{\prime}\right), \\
p_{k+1} & =p_{k}+\sigma_{p}^{2}, \\
G & =\frac{p_{k}^{\prime}}{p_{k}^{\prime}+\sigma_{m}^{2}}, \\
p_{k} & =p_{k}^{\prime}(1-G),
\end{aligned}
$$

where $\hat{x}_{k}, \hat{x}_{k}^{\prime}, G, z_{k}, p_{k}, p_{k}^{\prime}, \sigma_{p}^{2}$, and $\sigma_{m}^{2}$ are an estimate of the state $x$ at time $k$, prior estimate of $\hat{x}_{k}$, Kalman gain, actual value of $x$ at time $k$, error covariance at time $k$, prior estimate of $p_{k}$, and the noise covariance of the prediction and measurement, respectively. The measurement and prediction errors are considered to be Gaussian. Figure 20 compares the time profiles of $\Delta T_{\mathrm{NV}}$ with the 20-point moving average and the Kalman filter with $\sigma_{p}^{2}=0.01$ and $\sigma_{m}^{2}=1$. The two types 


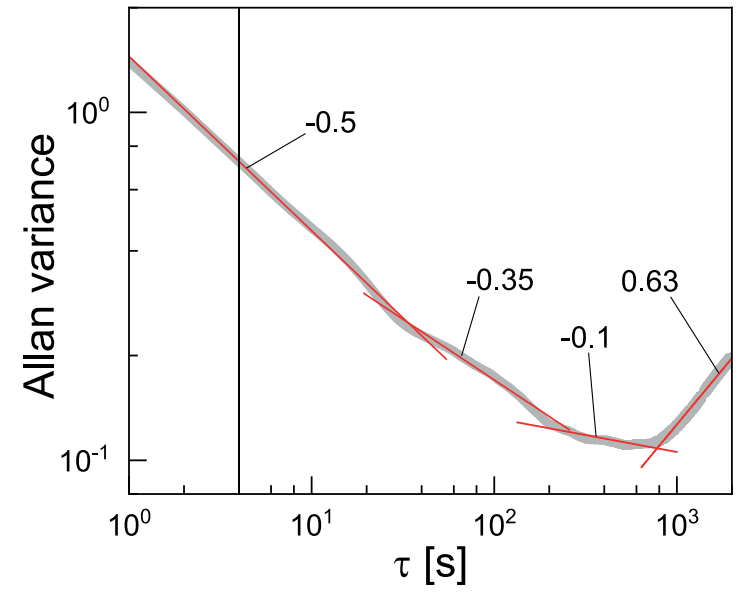

FIG. 23. Linear slopes in the Allan variance data of Fig. 9(b). Slopes of the distinct four regions are indicated. A tracking time of $4 \mathrm{~s}$ is indicated by the vertical solid line.

of filters effectively extract the dynamics of temperature in the ND quantum thermometry and match each other. While the 20-point moving average filter is sufficient to extract the temperature dynamics if it is working on this type of temperature change step, the successful implementation of the Kalman filter should be useful for more realistic transient temperature dynamics that are not easily predicted. Furthermore, a recent demonstration of the active feedback quantum thermometry indicated that by coupling it with a magnetic field may harness the rapid estimation protocol of the Kalman filter. (a)

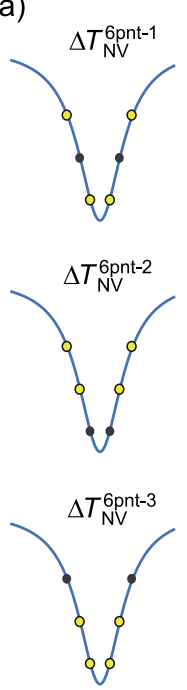

(b)

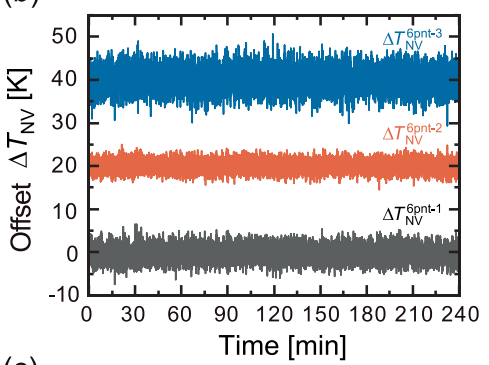

(c)

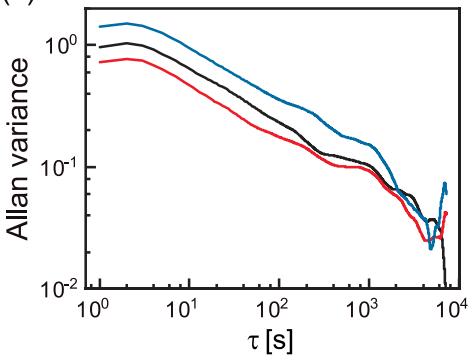

FIG. 24. Supplementary data of the Allan variance of the 6point method. (a) The three types of the 4-point configurations are schematically depicted. (b) Time profiles of $\Delta T_{\mathrm{NV}}$ with an offset of $20 \mathrm{~K}$. (c) Allan variance profile of the respective $\Delta T_{\mathrm{NV}}$.
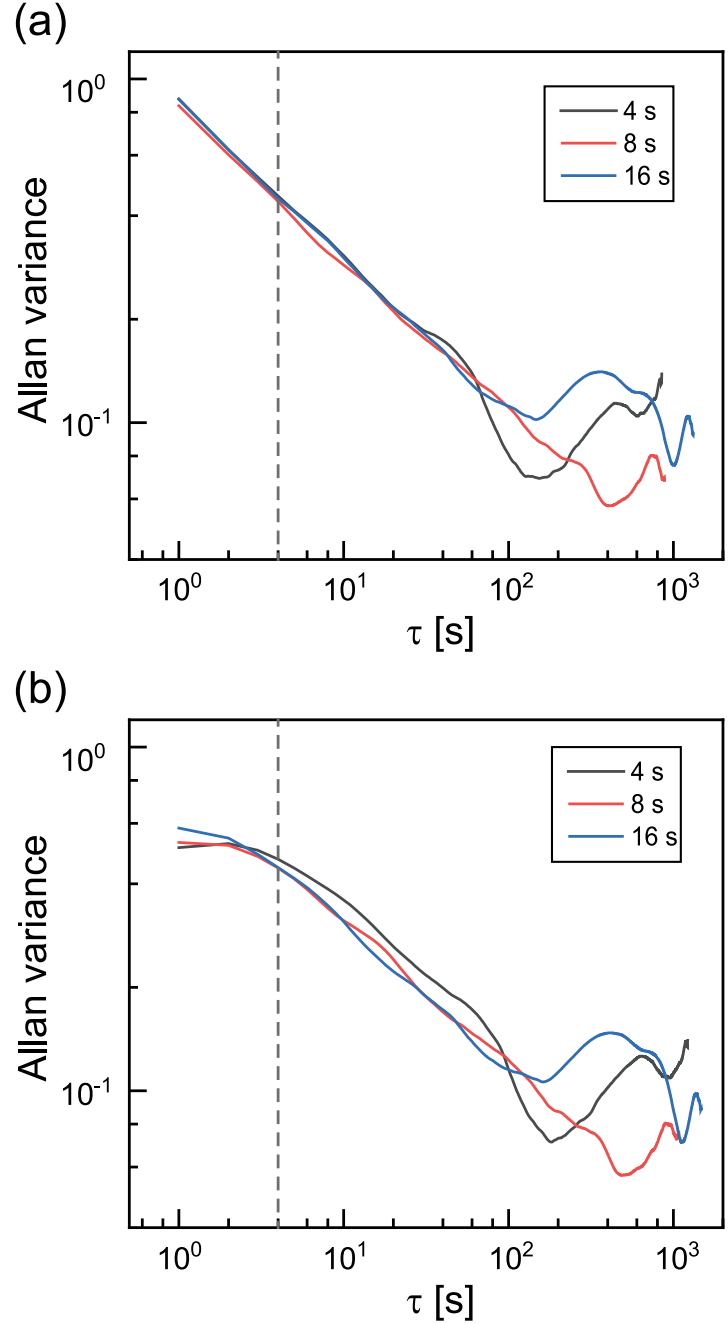

FIG. 25. Allan variance profiles in the 4-point method with the tracking period of 4,8 , and $16 \mathrm{~s}$ for the two types of measurements in which the tracking times are (a) ignored or (b) included via interpolation (b).

\section{APPENDIX J: TEMPERATURE DEPENDENCE OF THE REFRACTIVE INDEX OF IMMERSION OIL AND POINT SPREAD FUNCTIONS}

Figure 21 presents the temperature dependence of the refractive index from the four major suppliers. The index of the Olympus immersion oil was monitored over $12 \mathrm{~h}$ at $20^{\circ} \mathrm{C}$. The index was measured using a temperature controllable refractometer (Abbemat 500, Anton Paar). The products used were 518F (444960, Zeiss), Type-F2 (MXA22192, Nikon), Type-F (11513859, Leica), and Type-F (IMMOIL-F, Olympus). The temperature dependence of the index can be linearly fitted as follows as shown in Table IV.

Figure 22 shows the PSFs in the present experimental setup at $T_{\mathrm{S}}=27,32$, and $37^{\circ} \mathrm{C}$. The PSFs were visualized by performing 3D scanning across the same single ND placed on the coverslip. As $T_{\mathrm{S}}$ increased, the PSF was elongated along the $Z$ direction, which may affect the optical throughput at the pinhole. However, we were not able to determine if this PSF change caused the temperature dependent variation of $I_{\text {tot }}$ because of the substantial noise included in the visualized 
PSFs and uncertainty of the exact size of spatial filtering at the face of optical fiber working as pinhole.

\section{APPENDIX K: DETAILED ANALYSIS OF ALLAN VARIANCE}

Figure 23 presents the slopes of the linear fit to each stage of the Allan variance data for the 4-point measurement of Fig. 9(b). For a more detailed understanding of the 6point analysis, the time profiles of $\Delta T_{\mathrm{NV}}^{6 \mathrm{pnt}-1}, \Delta T_{\mathrm{NV}}^{6 \mathrm{pnt}-2}$, and $\Delta T_{\mathrm{NV}}^{6 \mathrm{pnt}-3}$ are plotted for the data shown in Fig. 24(c). The noise level varies for the three types of $\Delta T_{\mathrm{NV}}^{6 \mathrm{pnt}} \cdot \Delta T_{\mathrm{NV}}^{6 \mathrm{pnt}-3}$ yields the smallest noise and the noise increased in the order of $\Delta T_{\mathrm{NV}}^{6 \mathrm{pnt}-1}$ and $\Delta T_{\mathrm{NV}}^{6 \mathrm{pnt}-2}$. The Allan variances of these three types are shown in Fig. 24(f). Although the magnitude of $\sigma(\tau)$ is different for these three types, their variance profiles are the same, which indicates that they share common noise sources. Note that the proposed 6-point method provides the same precision compared to the 4-point method because of the estimation function for the frequency shift of the ODMR line. Rather, the 6-point method is used to understand the experi- mental hardware necessary for the multipoint measurements. In principle, the 6-point method provides more information than the 4-point analysis and may be useful to estimate the change of the whole spectral shape of the ODMR if designated equations are used.

The effect of the positional tracking period on the Allan variance profiles in the 4-point measurements was also investigated. In the main text, the positional tracking is performed every $t_{\text {track }}=4 \mathrm{~s}$. This tracking time can be varied if the positional drift is sufficiently small during the tracking period. Figures 25(a) and 25(b) present the Allan variance profiles of the thermometry stability data for $t_{\text {track }}=4,8$, $16 \mathrm{~s}$ where the interval time is included by interpolation, and ignored to be considered in the 1-s constant sampling time, respectively. Note that they all exhibit a flat noise profile until $4 \mathrm{~s}$, regardless of the tracking period, as shown in Fig. 25(a); when ignoring the tracking time, they present approximately the same profile until $80 \mathrm{~s}$, as shown in Fig. 25(b). The noise profiles between 4 and $80 \mathrm{~s}$ differ when including the tracking period via interpolation, as shown in Fig. 25(a). These results indicate that the first plateau of the noise profile until $4 \mathrm{~s}$ is due to the uneven time spacing of the data in the Allan variance calculation or the interpolation effect.
[1] J. R. Maze, P. L. Stanwix, J. S. Hodges, S. Hong, J. M. Taylor, P. Cappellaro, L. Jiang, M. V. Gurudev Dutt, E. Togan, A. S. Zibrov et al., Nanoscale magnetic sensing with an individual electronic spin in diamond, Nature (London) 455, 644 (2008).

[2] R. Schirhagl, K. Chang, M. Loretz, and C. L. Degen, Nitrogenvacancy centers in diamond: Nanoscale sensors for physics and biology, Annu. Rev. Phys. Chem. 65, 83 (2014).

[3] C. L. Degen, F. Reinhard, and P. Cappellaro, Quantum sensing, Rev. Mod. Phys. 89, 035002 (2017).

[4] G. Petrini, E. Moreva, E. Bernardi, P. Traina, G. Tomagra, V. Carabelli, I. P. Degiovanni, and M. Genovese, Is a quantum biosensing revolution approaching? Perspectives in NV-assisted current and thermal biosensing in living cells, Adv. Quantum Technol. 2000066 (2020).

[5] D. R. Glenn, R. R. Fu, P. Kehayias, D. Le Sage, E. A. Lima, B. P. Weiss, and R. L. Walsworth, Micrometer-scale magnetic imaging of geological samples using a quantum diamond microscope, Geochem. Geophys. Geosys. 18, 3254 (2017).

[6] T. Rendler, Fluorescent nanodiamonds as a sensor and life science probe, Ph.D. thesis, University of Stuttgart, 2018.

[7] E. V. Levine, M. J. Turner, P. Kehayias, C. A. Hart, N. Langellier, R. Trubko, D. R. Glenn, R. R. Fu, and R. L. Walsworth, Principles and techniques of the quantum diamond microscope, Nanophotonics 8, 1945 (2019).

[8] M. Lesik, T. Plisson, L. Toraille, J. Renaud, F. Occelli, M. Schmidt, O. Salord, A. Delobbe, T. Debuisschert, L. Rondin, P. Loubeyre, and J.-F. Roch, Magnetic measurements on micrometer-sized samples under high pressure using designed NV centers, Science 366, 1359 (2019).

[9] S. Choe, J. Yoon, M. Lee, J. Oh, D. Lee, H. Kang, Chul-Ho Lee, and D. Lee, Precise temperature sensing with nanoscale thermal sensors based on diamond NV centers, Curr. Appl. Phys. 18, 1066 (2018).
[10] P. Andrich, J. Li, X. Liu, F. J. Heremans, P. F. Nealey, and D. D. Awschalom, Microscale-resolution thermal mapping using a flexible platform of patterned quantum sensors, Nano Lett. 18, 4684 (2018).

[11] R. S. Schoenfeld and W. Harneit, Real Time Magnetic Field Sensing and Imaging Using a sIngle Spin in Diamond, Phys. Rev. Lett. 106, 030802 (2011).

[12] C. Bonato, M. S. Blok, H. T. Dinani, D. W. Berry, M. L. Markham, D. J. Twitchen, and R. Hanson, Optimized quantum sensing with a single electron spin using real-time adaptive measurements, Nat. Nanotech. 11, 247 (2016).

[13] T. Rendler, J. Neburkova, O. Zemek, J. Kotek, A. Zappe, Z. Chu, P. Cigler, and J. Wrachtrup, Optical imaging of localized chemical events using programmable diamond quantum nanosensors, Nat. Commun. 8, 14701 (2019).

[14] A. M. Wojciechowski, M. Karadas, A. Huck, C. Osterkamp, S. Jankuhn, J. Meijer, F. Jelezko, and U. L. Andersen, Contributed review: Camera-limits for wide-field magnetic resonance imaging with a nitrogen-vacancy spin sensor, Rev. Sci. Instrum. 89, 031501 (2018).

[15] J. M. Schloss, J. F. Barry, M. J. Turner, and R. L. Walsworth, Simultaneous Broadband Vector Magnetometry Using Solid-State Spins, Phys. Rev. Appl. 10, 034044 (2018).

[16] K. Ambal and R. D. McMichael, A differential rate meter for real-time peak tracking in optically detected magnetic resonance at low photon count rates, Rev. Sci. Instrum. 90, 023907 (2019).

[17] J. A. Akin, Homeostatic processes for thermoregulation, Nat. Ed. Knowl. 3, 7 (2011).

[18] C. W. Meyer, Youichirou Ootsuka, and A. A. Romanovsky, Body temperature measurements for metabolic phenotyping in mice, Front. Physiol. 8, 520 (2017). 
[19] M. L. Begasse, M. Leaver, F. Vazquez, S. W. Grill, and A. A. Hyman, Temperature dependence of cell division timing accounts for a shift in the thermal limits of $C$. elegans and $C$. briggsae, Cell Rep. 10, 647 (2015).

[20] J. Chong, C. Amourda, and T. E. Saunders, Temporal development of drosophila embryos is highly robust across a wide temperature range, J. R. Soc. Interface 15, 20180304 (2018).

[21] G. Kucsko, P. C. Maurer, N. Y. Yao, M. Kubo, H. J. Noh, P. K. Lo, H. Park, and M. D. Lukin, Nanometre-scale thermometry in a living cell, Nature (London) 500, 54 (2013).

[22] D. A. Simpson, E. Morrisroe, J. M. McCoey, A. H. Lombard, D. C. Mendis, F. Treussart, L. T. Hall, S. Petrou, and L. C. L. Hollenberg, Non-neurotoxic nanodiamond probes for intraneuronal temperature mapping, ACS Nano 11, 12077 (2017).

[23] H. Yukawa, M. Fujiwara, K. Kobayashi, Y. Kumon, K. Miyaji, Y. Nishimura, K. Oshimi, Y. Umehara, Y. Teki, T. Iwasaki et al., A quantum thermometric sensing and analysis system using fluorescent nanodiamonds for the evaluation of living stem cell functions according to intracellular temperature, Nanoscale Adv. 2, 1859 (2020).

[24] T. Sekiguchi, S. Sotoma, and Y. Harada, Fluorescent nanodiamonds as a robust temperature sensor inside a single cell, Biophys. Physicobiol. 15, 229 (2018).

[25] J. Choi, H. Zhou, R. Landig, H.-Y. Wu, X. Yu, S. E. Von Stetina, G. Kucsko, S. E. Mango, D. J. Needleman, A. D. T. Samuel et al., Probing and manipulating embryogenesis via nanoscale thermometry and temperature control, Proc. Natl. Acad. Sci. U. S. A. 117, 14636 (2020).

[26] M. Fujiwara, S. Sun, A. Dohms, Y. Nishimura, K. Suto, Y. Takezawa, K. Oshimi, L. Zhao, N. Sadzak, Y. Umehara et al., Real-time nanodiamond thermometry probing in vivo thermogenic responses, Sci. Adv. 6, eaba9636 (2020).

[27] P. Neumann, I. Jakobi, F. Dolde, C. Burk, R. Reuter, G. Waldherr, J. Honert, T. Wolf, A. Brunner, J. H. Shim et al., High-precision nanoscale temperature sensing using single defects in diamond, Nano Lett. 13, 2738 (2013).

[28] D. M. Toyli, C. F. de las Casas, D. J. Christle, V. V. Dobrovitski, and D. D. Awschalom, Fluorescence thermometry enhanced by the quantum coherence of single spins in diamond, Proc. Natl. Acad. Sci. U. S. A. 110, 8417 (2013).

[29] Y.-K. Tzeng, P.-C. Tsai, H.-Y. Liu, O. Y. Chen, H. Hsu, F.-G. Yee, M.-S. Chang, and H.-C. Chang, Time-resolved luminescence nanothermometry with nitrogen-vacancy centers in nanodiamonds, Nano Lett. 15, 3945 (2015).

[30] H. Clevenson, M. E. Trusheim, C. Teale, T. Schröder, D. Braje, and D. Englund, Broadband magnetometry and temperature sensing with a light-trapping diamond waveguide, Nat. Phys. 11, 393 (2015).

[31] J. Wang, F. Feng, J. Zhang, J. Chen, Z. Zheng, L. Guo, W. Zhang, X. Song, G. Guo, L. Fan et al., High-sensitivity temperature sensing using an implanted single nitrogen-vacancy center array in diamond, Phys. Rev. B 91, 155404 (2015).

[32] N. Wang, G.-Q. Liu, W.-H. Leong, H. Zeng, X. Feng, S.-H. Li, F. Dolde, H. Fedder, J. Wrachtrup, X.-D. Cui et al., Magnetic Criticality Enhanced Hybrid Nanodiamond Thermometer under Ambient Conditions, Phys. Rev. X 8, 011042 (2018).

[33] G.-Q. Liu, Xi Feng, N. Wang, Q. Li, and R.-B. Liu, Coherent quantum control of nitrogen-vacancy center spins near 1000 kelvin, Nat. Commun. 10, 1 (2019).
[34] C.-F. Liu, W.-H. Leong, K. Xia, Xi Feng, A. Finkler, A. Denisenko, J. Wrachtrup, Q. Li, and R.-B. Liu, Ultra-sensitive hybrid diamond nanothermometer, Natl. Sci. Rev. (2020) nwaa194.

[35] V. M. Acosta, E. Bauch, M. P. Ledbetter, A. Waxman, L.-S. Bouchard, and D. Budker, Temperature Dependence of the Nitrogen-Vacancy Magnetic Resonance in Diamond, Phys. Rev. Lett. 104, 070801 (2010).

[36] X.-D. Chen, C.-H. Dong, F.-W. Sun, C.-L. Zou, J.-M. Cui, Z.-F Han, and G.-C. Guo, Temperature dependent energy level shifts of nitrogen-vacancy centers in diamond, Appl. Phys. Lett. 99, 161903 (2011).

[37] M. W. Doherty, V. M. Acosta, A. Jarmola, M. S. J. Barson, N. B. Manson, D. Budker, and L. C. L. Hollenberg, Temperature shifts of the resonances of the $\mathrm{NV}^{-}$center in diamond, Phys. Rev. B 90, 041201(R) (2014).

[38] K. Hayashi, Y. Matsuzaki, T. Taniguchi, T. Shimo-Oka, I. Nakamura, S. Onoda, T. Ohshima, H. Morishita, M. Fujiwara, S. Saito, and N. Mizuochi, Optimization of Temperature Sensitivity Using the Optically Detected Magnetic-Resonance Spectrum of a Nitrogen-Vacancy Center Ensemble, Phys. Rev. Appl. 10, 034009 (2018).

[39] Y. Matsuzaki, H. Morishita, T. Shimooka, T. Tashima, K. Kakuyanagi, K. Semba, W. J. Munro, H. Yamaguchi, N. Mizuochi, and S. Saito, Optically detected magnetic resonance of high-density ensemble of $\mathrm{NV}^{-}$centers in diamond, J. Phys. Condens. Matter 28, 275302 (2016).

[40] M. Fujiwara, K. Yoshida, T. Noda, H. Takashima, A. W. Schell, N. Mizuochi, and S. Takeuchi, Manipulation of single nanodiamonds to ultrathin fiber-taper nanofibers and control of NV-spin states toward fiber-integrated $\lambda$-systems, Nanotechnology 27, 455202 (2016).

[41] A. Dréau, M. Lesik, L. Rondin, P. Spinicelli, O. Arcizet, J.-F. Roch, and V. Jacques, Avoiding power broadening in optically detected magnetic resonance of single NV defects for enhanced dc magnetic field sensitivity, Phys. Rev. B 84, 195204 (2011).

[42] M. Fujiwara, Y. Shikano, R. Tsukahara, S. Shikata, and H. Hashimoto, Observation of the linewidth broadening of single spins in diamond nanoparticles in aqueous fluid and its relation to the rotational Brownian motion, Sci. Rep. 8, 14773 (2018).

[43] H. Clevenson, E. H. Chen, F. Dolde, C. Teale, D. Englund, and D. Braje, Diamond-nitrogen-vacancy electronic and nuclear spin-state anticrossings under weak transverse magnetic fields, Phys. Rev. A 94, 021401(R) (2016).

[44] J. P. Tetienne, L. Rondin, P. Spinicelli, M. Chipaux, T. Debuisschert, J. F. Roch, and V. Jacques, Magnetic-fielddependent photodynamics of single NV defects in diamond: An application to qualitative all-optical magnetic imaging, New J. Phys. 14, 103033 (2012).

[45] C. Foy, L. Zhang, M. E. Trusheim, K. R. Bagnall, M. Walsh, E. N. Wang, and D. R. Englund, Wide-field magnetic field and temperature imaging using nanoscale quantum sensors, ACS Appl. Mater. Interf. 12, 26525 (2020).

[46] K. Seng, Y. Chen, K. M. A. Chai, T. Wang, D. C. Y. Fun, Y. S. Teo, P. M. S. Tan, W. H. Ang, and J. K. W. Lee, Tracking body core temperature in military thermal environments: An extended Kalman filter approach, in 2016 IEEE 13th International Conference on Wearable and Implantable Body Sensor Networks (BSN) (IEEE, San Francisco, CA, 2016), pp. 296299. 
[47] T. Plakhotnik and D. Gruber, Luminescence of nitrogenvacancy centers in nanodiamonds at temperatures between 300 and $700 \mathrm{~K}$ : Perspectives on nanothermometry, Phys. Chem. Chem. Phys. 12, 9751 (2010).

[48] J. C. Price, S. J. Levett, V. Radu, D. A. Simpson, A. M. Barcons, C. F. Adams, and M. L. Mather, Quantum sensing in a physiological-like cell niche using fluorescent nanodiamonds embedded in electrospun polymer nanofibers, Small 15, 1900455 (2019).

[49] S. Kaufmann, D. A. Simpson, L. T. Hall, V. Perunicic, P. Senn, S. Steinert, L. P. McGuinness, B. C. Johnson, T. Ohshima, F. Caruso et al., Detection of atomic spin labels in a lipid bilayer using a single-spin nanodiamond probe, Proc. Natl. Acad. Sci. U. S. A. 110, 10894 (2013).

[50] S. Sotoma, F.-J. Hsieh, Y.-W. Chen, P.-C. Tsai, and H.-C. Chang, Highly stable lipid-encapsulation of fluorescent nanodiamonds for bioimaging applications, Chem. Commun. 54, 1000 (2018).

[51] S. Claveau, J.-R. Bertrand, and F. Treussart, Fluorescent nanodiamond applications for cellular process sensing and cell tracking, Micromachines 9, 247 (2018).

[52] N. Mohan, C.-S. Chen, H.-H. Hsieh, Yi-Chun Wu, and H.-C. Chang, In vivo imaging and toxicity assessments of fluorescent nanodiamonds in Caenorhabditis elegans, Nano Lett. 10, 3692 (2010).

[53] D. A. Simpson, A. J. Thompson, M. Kowarsky, N. F. Zeeshan, M. S. J. Barson, L. T. Hall, Y. Yan, S. Kaufmann, B. C. Johnson, T. Ohshima et al., In vivo imaging and tracking of individual nanodiamonds in Drosophila melanogaster embryos, Biomed. Opt. Express 5, 1250 (2014).

[54] Y.-C. Lin, K.-T. Wu, Z.-R. Lin, E. Perevedentseva, A. Karmenyan, M.-D. Lin, and C.-L. Cheng, Nanodiamond for biolabelling and toxicity evaluation in the zebrafish embryo in vivo, J. Biophoton. 9, 827 (2016).

[55] M. W. Doherty, V. V. Struzhkin, D. A. Simpson, L. P. McGuinness, Y. Meng, A. Stacey, T. J. Karle, R. J. Hemley, N. B. Manson, L. C. L. Hollenberg, and S. Prawer, Electronic Properties and Metrology Applications of the Diamond $\mathrm{NV}^{-}$Center under Pressure, Phys. Rev. Lett. 112, 047601 (2014).

[56] J. Cai, F. Jelezko, and M. B. Plenio, Hybrid sensors based on colour centres in diamond and piezoactive layers, Nat. Commun. 5, 4065 (2014).

[57] F. Dolde, H. Fedder, M. W. Doherty, T. Nöbauer, F. Rempp, G. Balasubramanian, T. Wolf, F. Reinhard, L. C. L. Hollenberg, F. Jelezko, and J. Wrachtrup, Electric-field sensing using single diamond spins, Nat. Phys. 7, 459 (2011).

[58] T. Iwasaki, W. Naruki, K. Tahara, T. Makino, H. Kato, M. Ogura, D. Takeuchi, S. Yamasaki, and M. Hatano, Direct nanoscale sensing of the internal electric field in operating semiconductor devices using single electron spins, ACS Nano 11, 1238 (2017).

[59] G. Reina, Li Zhao, A. Bianco, and N. Komatsu, Chemical functionalization of nanodiamonds: Opportunities and challenges ahead, Angew. Chem. Int. Ed. 58, 17918 (2019).

[60] S. Sotoma, R. Igarashi, J. Iimura, Y. Kumiya, H. Tochio, Y. Harada, and M. Shirakawa, Suppression of nonspecific protein-nanodiamond adsorption enabling specific targeting of nanodiamonds to biomolecules of interest, Chem. Lett. 44, 354 (2015).
[61] C.-L. Lin, C.-H. Lin, H.-C. Chang, and M.-C. Su, Protein attachment on nanodiamonds, J. Phys. Chem. A 119, 7704 (2015).

[62] T. Zhang, G.-Q. Liu, W.-H. Leong, C.-F. Liu, M.-H. Kwok, To Ngai, R.-B. Liu, and Q. Li, Hybrid nanodiamond quantum sensors enabled by volume phase transitions of hydrogels, Nat. Commun. 9, 3188 (2018).

[63] H. Raabova, D. Chvatil, and P. Cigler, Diamond nanooptode for fluorescent measurements of $\mathrm{pH}$ and temperature Nanoscale 11, 18537 (2019).

[64] M. Fujiwara, R. Tsukahara, Y. Sera, H. Yukawa, Y. Baba, S. Shikata, and H. Hashimoto, Monitoring spin coherence of single nitrogen-vacancy centers in nanodiamonds during $\mathrm{pH}$ changes in aqueous buffer solutions, RSC Adv. 9, 12606 (2019).

[65] T. Fujisaku, R. Tanabe, S. Onoda, R. Kubota, T. F. Segawa, F. T.-K. So, T. Ohshima, I. Hamachi, M. Shirakawa, and R. Igarashi, $\mathrm{pH}$ nanosensor using electronic spins in diamond, ACS Nano 13, 11726 (2019).

[66] A. Sigaeva, Y. Ong, V. G. Damle, A. Morita, K. J. van der Laan, and R. Schirhagl, Optical detection of intracellular quantities using nanoscale technologies, Acc. Chem. Res. 52, 1739 (2019).

[67] A. Ermakova, G. Pramanik, J.-M. Cai, G. Algara-Siller, U. Kaiser, T. Weil, Y.-K. Tzeng, H.-Ch. Chang, L. P. McGuinness, M. B. Plenio et al., Detection of a few metallo-protein molecules using color centers in nanodiamonds, Nano Lett. 13, 3305 (2013).

[68] W. W.-W. Hsiao, Y. Y. Hui, P.-C. Tsai, and H.-C. Chang, Fluorescent nanodiamond: A versatile tool for long-term cell tracking, super-resolution imaging, and nanoscale temperature sensing, Acc. Chem. Res. 49, 400 (2016).

[69] I.-S. Woo, I.-K. Rhee, and H.-D. Park, Differential damage in bacterial cells by microwave radiation on the basis of cell wall structure, Appl. Environ. Microbiol. 66, 2243 (2000).

[70] S. Karaveli, O. Gaathon, A. Wolcott, R. Sakakibara, O. A Shemesh, D. S. Peterka, E. S. Boyden, J. S. Owen, R. Yuste, and D. Englund, Modulation of nitrogen vacancy charge state and fluorescence in nanodiamonds using electrochemical potential, Proc. Natl. Acad. Sci. U. S. A. 113, 3938 (2016).

[71] T. Plakhotnik, M. W. Doherty, J. H. Cole, R. Chapman, and N. B. Manson, All-optical thermometry and thermal properties of the optically detected spin resonances of the $\mathrm{NV}^{-}$center in nanodiamond, Nano Lett. 14, 4989 (2014).

[72] T. Plakhotnik, H. Aman, and H.-C. Chang, All-optical singlenanoparticle ratiometric thermometry with a noise floor of 0.3 $\mathrm{K} \mathrm{Hz}^{-1 / 2}$, Nanotechnology 26, 245501 (2015).

[73] M. Fukami, C.G. Yale, P. Andrich, X. Liu, F.J. Heremans, P.F. Nealey, and D.D. Awschalom, All-optical cryogenic thermometry based on nitrogen-vacancy centers in nanodiamonds, Phys. Rev. Appl. 12, 014042 (2019).

[74] K. Okabe, N. Inada, C. Gota, Y. Harada, T. Funatsu, and S. Uchiyama, Intracellular temperature mapping with a fluorescent polymeric thermometer and fluorescence lifetime imaging microscopy, Nat. Commun. 3, 705 (2012).

[75] T. Sugimura, S. Kajimoto, and T. Nakabayashi, Label-free imaging of intracellular temperature by using the O-H stretching Raman band of water, Angew. Chem. Int. Ed. 59, 7755 (2020). 
[76] T. Tsuji, K. Ikado, H. Koizumi, S. Uchiyama, and K. Kajimoto, Difference in intracellular temperature rise between matured and precursor brown adipocytes in response to uncoupler and $\beta$-adrenergic agonist stimuli, Sci. Rep. 7, 12889 (2017).

[77] X. Qiu, Q. Zhou, X. Zhu, Z. Wu, W. Feng, and F. Li, Ratiometric upconversion nanothermometry with dual emission at the same wavelength decoded via a time-resolved technique, Nat. Commun. 11, 41 (2020).
[78] D. Jaque and F. Vetrone, Luminescence nanothermometry, Nanoscale 4, 4301 (2012).

[79] B. del Rosal, D. Ruiz, I. Chaves-Coira, B. H. Juárez, L. Monge, G. Hong, N. Fernández, and D. Jaque, In vivo contactless brain nanothermometry, Adv. Funct. Mater. 28, 1806088 (2018).

[80] W. T. Higgins, A comparison of complementary and Kalman filtering, IEEE Trans. Aerospace Elec. Syst. AES-11, 321 (1975). 